\title{
ACCOUNTING FOR MAGNETIC SATURATION IN INDUCTION MACHINES MODELLING
}

\author{
H. OUADI ${ }^{1}$, F. GIRI ${ }^{1, *}$, L. DUGARD ${ }^{2}$ \\ ${ }^{1}$ GREYC, University of Caen, Caen, France \\ ${ }^{2}$ GIPSA Lab, INPG, Grenoble, France \\ * Corresponding author: giri@greyc.ensicaen.fr
}

\begin{abstract}
A new model is developed for (uniform air-gap) induction motor that accounts for the saturation feature of the machine magnetic circuit. It is built-up starting from the basic electrical/mechanical laws and turns out to be quite different from the standard model that is widely used in control oriented literature. Specifically, the new model involves state-dependent parameters. An experimental validation using a real $7.5 \mathrm{KW}$ machine proved the good accuracy of the proposed model. A simpler control-oriented version of the model is also developed and shown to be quite representative of the machine.
\end{abstract}

\section{Introduction}

It is widely recognized that induction motor is going to become the main actuator for industrial purposes. Indeed, as compared to the DC machine, it provides a better power/mass ratio, allows a simpler maintenance (as it includes no mechanical commutators) and costs relatively cheaper. However, the problem of controlling induction motor is more complex because of its multivariable and nonlinear nature. Besides, some of its state variables are not accessible to measurement. The problem of induction motor control and observation has been given a great deal of interest over the last decade see e.g. [1]-[2]. However, most of previous works have been based on the standard model where the magnetic characteristic is described by a linear relation. As a matter of fact, such a characteristic is nonlinear in physical machines: it exhibits saturation and hysteresis features. To avoid drastic control performance deterioration, due to magnetic circuit saturation, special precautions should be taken when it comes to practical implementation of the obtained controllers. Specifically, two implementation strategies are generally followed to avoid control performance deterioration due to magnetic saturation. The first strategy consists in constraining the rotor flux to take small values so that the machine operates in the linear zone of its magnetic characteristic i.e. the domain where the standard model is representative of the machine. The drawback of such a solution is that the machine does not operate in optimal efficiency conditions especially in presence of high loads. Then, the consumed stator current is unnecessary large. The second control strategy consists in regulating the rotor flux norm around its nominal value which is generally located at the nonlinear part of the magnetic characteristic. To meet this objective, the parameters of the standard model (based upon when designing the controller) should be tuned so that they correspond to the above nominal operation point. Then, the machine efficiency is maximal when the machine load torque is close to its nominal value. But, the load is usually subject to large variations in practical applications. 
If the load torque is small (with respect to the nominal load), there is a useless energy stored in stator inductances reducing the machine efficiency.

To achieve high-performance varying-speed operation mode for induction machines, it is necessary to use controllers that allow large flux variations. Indeed, allowing large flux variations makes possible the achievement of a suitable power factor and a high efficiency (by limiting current absorption). But, in order to allow large flux variations the controller must be developed by using a model that takes into account the nonlinear nature of the machine magnetic characteristic. The question is: how such model can be obtained?

The present work precisely focuses on such a modelling issue. This has been coped with, in [3]-[4], [9]-[10] by just letting the mutual inductance coefficient associated with a given rotor flux direction ( $d$ or $q$ ) be a nonlinear function of the stator current along the same direction. This assumption is not realistic because it amounts to neglect the crosssaturation feature. Furthermore, neither the nonlinear functions approximating the mutual inductances nor the resulting (saturated) model are explicitly described in the mentioned works. A quite different approach to account for the saturation feature has been proposed for synchronous machines using energetic considerations [12]-[13]. The obtained model is expressed in the complex notation which makes it only valid in steady-state harmonic regimes.

The present paper develops a new approach that appropriately accounts for the magnetic saturation phenomenon in induction machine modelling. It thoroughly contrasts with the previous approaches which heavily relied upon the standard unsaturated model. Presently, the starting point is the machine electric equivalent scheme where the stator and the rotor are represented by triphase coils [7]. Additional physical laws will be applied to account for the (well known) coupling that exists (even in the case of a uniform air-gap machine) between both axes of an AC-machine, [12]. Such a coupling (usually called cross-saturation), is due to the nonlinear properties of magnetic materials. As suggested in [5], the magnetic characteristic can be approximated by a nonlinear function that could be polynomial, exponential, arctangent, etc. Such a function links the air-gap flux $\Phi_{\mu}$ to the magnetizing current $I_{\mu}$ (this includes the contribution of both stator and rotor currents). The obtained model is experimentally validated using a 7.5 KW AC-machine. The input signals are chosen so that the machine operates both in the linear and nonlinear parts of the corresponding magnetic characteristic. The resulting responses of the new model turn out to be sufficiently close to those of the true machine. On the contrary, the standard model responses are not so close, especially when the machine operates in the saturation part. The new model is quite suitable for the machine simulation but can hardly be used in control design, because it is highly nonlinear and involves many parameters depending on the machine state variables. Therefore, a simpler version is developed by gathering all magnetic leakage inductances at the stator side. The 
simplified model proves to be quite accurate and, due to its simplicity, convenient for designing controllers and observers.

The paper is organized as follows: Section II is devoted to modelling the magnetic characteristic of the studied machine; the remaining electromechanical equations are established in Section III; in Section IV, the static magnetisation parameter is introduced and, in Section V, machine modelling is completed by establishing its state-space representation; an experimental validation of the obtained model as well as a comparison with the standard model are performed in Section VI. In section VII, a simplified version of the above model is derived.

Table 1. Notations

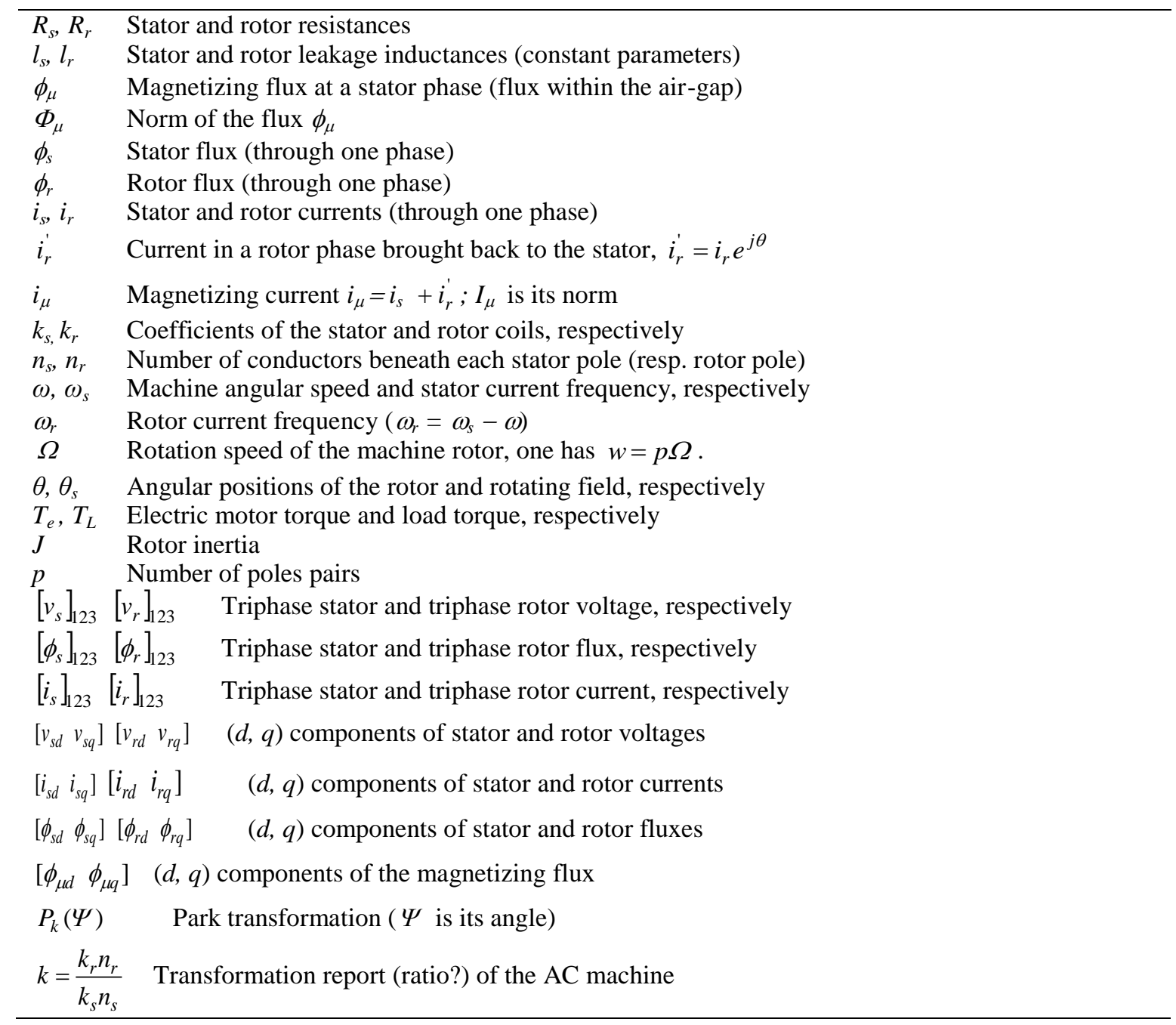




\section{Characterisation of magnetic saturation in AC machines}

The nonlinear feature of the magnetic circuit in induction motors has been accounted for in many ways. Early solutions suggested capturing this through nonlinear approximations of the $(B, H)$ characteristic, where $B$ denotes the magnetic field and $H$ the magnetic induction ([7], [11]). In more recent works, the magnetic saturation is accounted for through a flux-current relation called magnetic characteristic. This links the magnetizing flux norm (i.e. the useful flux at a stator phase) to the magnetizing current ([12]). Several laws have been suggested to describe the magnetic characteristic, e.g.:

$$
\Phi_{\mu}=\alpha \beta^{I_{\mu}} I_{\mu}^{\gamma} \quad \text { or } \quad I_{\mu}=\frac{s_{1}-s_{2}}{\left(b^{-n}+\Phi_{\mu}^{-n}\right)^{1 / n}}+s_{2} \Phi_{\mu} .
$$

Other functions such as arctangent or hyperbolic-tangent are also resorted to approximate the magnetic characteristic. In the present work, a spline polynomial approximation is developed to approach the nonlinearity of the magnetic circuit. This has several advantages such as: (i) simplicity of experimental determination, (ii) the larger the polynomial degree the better the approximation accuracy, (iii) simplicity of algebraic and differential transformations.

Let the (unknown) real magnetic characteristic be denoted $I_{\mu}=\lambda\left(\Phi_{\mu}\right)$. Our purpose is to build up a polynomial approximation for $\lambda\left(I_{\mu}\right)$. The first step consists in obtaining experimentally a set of points of the real machine characteristic. A spline interpolation of the experimental points is then performed, using suitable tools, to get a polynomial approximation, denoted $P($.) of the unknown function $\lambda($.). The larger the degree of $P($.$) ,$ the more smooth and accurate the approximation. Fig. 1 shows the polynomial $P($. obtained with $n=8$. In the sequel, we will also need an approximation of the inverse characteristic $I_{\mu}=\lambda^{-1}\left(\Phi_{\mu}\right)$. A polynomial approximation $P_{i n v}($.$) is obtained directly from$ the available experimental set of points, using the same tools as previously (Fig 2).

Remark 1. Fig. 1 shows that the largest linear zone of the magnetic characteristic corresponds to small values of the flux $\left(\Phi_{\mu}<0.7 \mathrm{wb}\right)$ whereas the machine nominal point, presently equal to $\Phi_{\mu}=0.9 \mathrm{~Wb}$, is located at the saturation elbow. 
Accounting for magnetic saturation in modelling induction machines

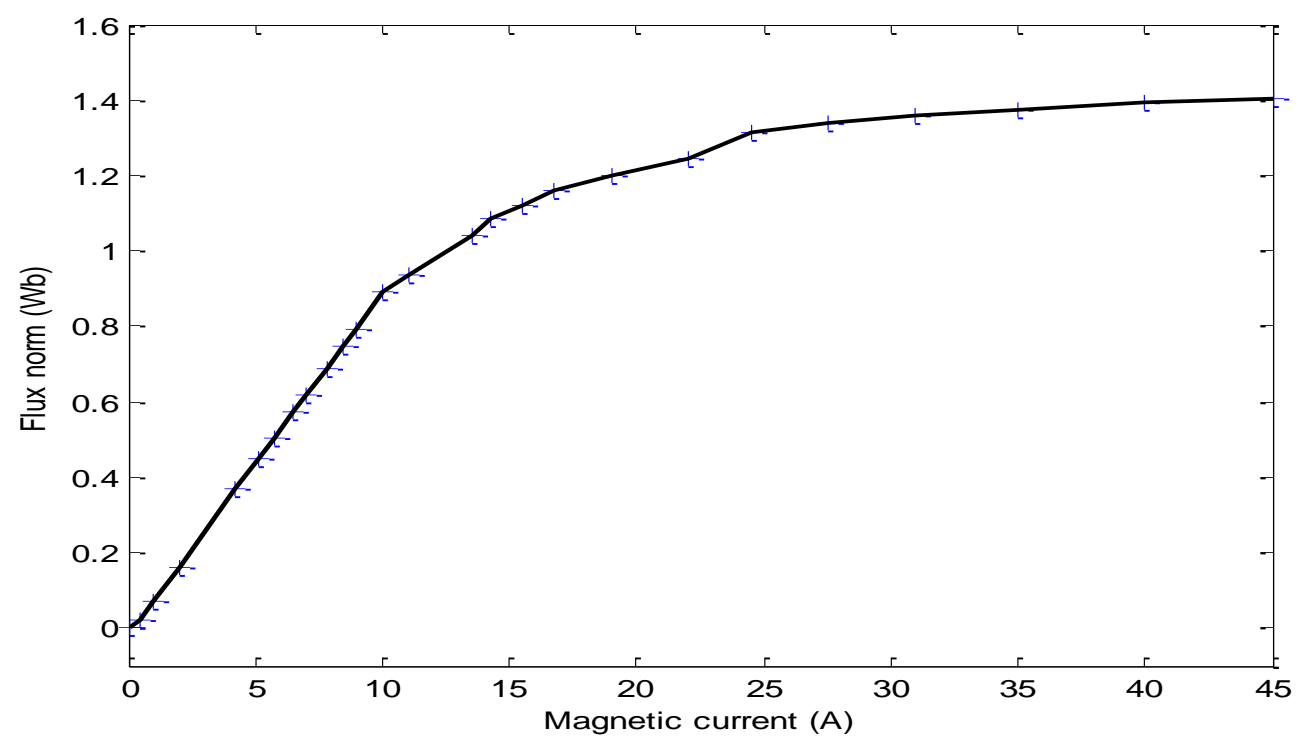

Fig 1. Magnetic characteristic $\Phi_{\mu}=\lambda\left(I_{\mu}\right)$. Crosses: experimental points $\left(\mathrm{I}_{\mu}, \Phi_{\mu}\right)$ Solid: interpolation $P($.$) . Unities: I_{\mu}(A), \Phi_{\mu}(W b)$

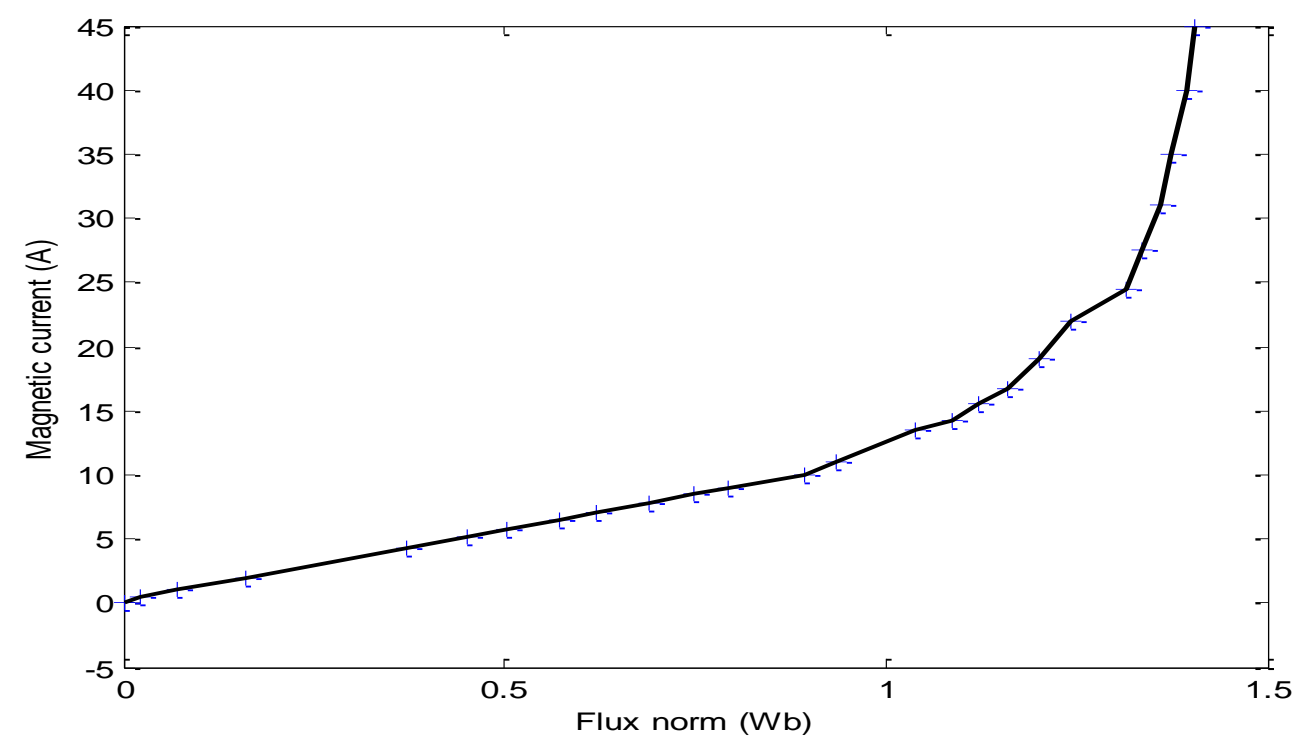

Fig 2: Inverse magnetic characteristic $I_{\mu}=\lambda^{-1}\left(\Phi_{\mu}\right)$. Crosses: experimental points $\left(\Phi_{\mu}, I_{\mu}\right)$. Solid: spline interpolation $P_{i n v}($.$) . Unities: I_{\mu}(A), \Phi_{\mu}(W b)$ 


\section{Induction machines equations}

\section{1 Park transformation of the stator and rotor voltages}

The proposed modelling approach is based on standard assumptions, namely: the machine is symmetrical, the air gap is smooth, the ferromagnetic losses are negligible, the induction distribution through the air gap is sinusoidal and all electromagnetic variables $\left(\left[\phi_{s}\right]_{1,2,3},\left[\phi_{r}\right]_{1,2,3},\left[i_{s}\right]_{1,2,3}, \ldots\right)$ define a well balanced triphase system, i.e. $\phi_{s 1}+\phi_{s 2}+\phi_{s 3}=0$. Applying the Park transformation to the triphase equations yields the following electrical equations, completed by the mechanical equation (see e.g. [7], [11]):

$$
\begin{aligned}
& V_{s d}=R_{s} i_{s d}+\frac{d}{d t} \phi_{s d}-\omega_{s} \phi_{s q} \\
& V_{s q}=R_{s} \cdot i_{s q}+\frac{d}{d t} \phi_{s q}+\omega_{s} \phi_{s d} \\
& 0=R_{r} i_{r d}+\frac{d}{d t} \phi_{r d}-\left(\omega_{s}-\omega\right) \phi_{r q} \\
& 0=R_{r} i_{r q}+\frac{d}{d t} \phi_{r q}+\left(\omega_{s}-\omega\right) \phi_{r d} \\
& \frac{d \Omega}{d t}=\frac{T_{e}}{J}-\frac{T_{L}}{J}-\frac{f}{J} \Omega
\end{aligned}
$$

\section{III.2 Saturated flux equations in the $(d, q)$ coordinates system}

.The magnetic fluxes for the stator and rotor phases are given by (see [7], [11]):

$$
\begin{aligned}
& \phi_{s}=\phi_{\text {leakage/st }}+\phi_{\mu}=l_{s} i_{s}+\phi_{\mu} \\
& \phi_{r}=\phi_{\text {leakage/ } r t}+\phi_{\mu r}=l_{r} i_{r}+\phi_{\mu r}
\end{aligned}
$$

where $\phi_{\mu}$ and $\phi_{\mu r}$ respectively denote the magnetizing air-gap fluxes along one phase of the stator and rotor; $\phi_{\text {leakage /st }}$ and $\phi_{\text {leakage/rt }}$ respectively denote the stator and rotor leakage fluxes. Equalities (6)-(7) mean that $\phi_{\text {leakage/st }}$ and $\phi_{\text {leakage/rt }}$ are respectively proportional to the stator and rotor currents. This is valid in real machines because the leakage flux, circulating in air, not in iron, is not large. Let $k$ denote the machine transformation ratio. The flux equations (6)-(7) become, in the $(d, q)$ coordinates:

$$
\begin{aligned}
& \phi_{s d}=l_{s} i_{s d}+\phi_{\mu d} \\
& \phi_{s q}=l_{s} i_{s q}+\phi_{\mu q} \\
& \phi_{r d}=l_{r} i_{r d}+k \phi_{\mu d} \\
& \phi_{r q}=l_{r} i_{r q}+k \phi_{\mu q}
\end{aligned}
$$


The contribution of the stator and rotor in the air-gap flux generation is expressed in term of the magnetizing current, denoted $i_{\mu}$ ([7], [12]). Therefore, the (d-q) components of the $\left[i_{\mu}\right]_{123}$ system satisfy:

$$
i_{\mu d}=i_{s d}+k i_{r d} \text { and } i_{\mu q}=i_{s q}+k i_{r q}
$$

The nonlinear feature of the machine magnetic characteristic is responsible for the crosssaturation effect, see ([12]-[13]). Accordingly, the component of a given (stator, rotor or magnetizing) flux, along a given axis ( $d$ or $q$ ), turns out to be dependent on both the $d$ and the $q$-components of both stator and rotor currents. Inspired from [5], the crosssaturation effect is accounted for letting the magnetizing flux be expressed as follows:

$$
\begin{aligned}
& \phi_{\mu d}=M_{d} i_{\mu d}+M_{d q} i_{\mu q}+\phi_{d 0} \\
& \phi_{\mu q}=M_{q} i_{\mu q}+M_{d q} i_{\mu d}+\phi_{q 0}
\end{aligned}
$$

where $M_{d}, M_{q}$ and $M_{d q}$ are new inductive parameters and:

. $i_{\mu d}, i_{\mu q}$ denote the $(d, q)$ components of the magnetizing current

. $M_{d} i_{\mu d}$ is the magnetizing flux generated, along the $d$-axis, by the components (along the same axis) of the stator and the rotor currents

. $M_{q} i_{\mu q}$ is the magnetizing flux generated, along the $q$-axis, by the components (along the same axis) of the stator and the rotor currents

. $M_{d q} i_{\mu d}$ is the coupling magnetizing flux generated, along the $q$-axis, by the components (along the same axis) of the stator and the rotor currents

. $M_{d q} i_{\mu q}$ is the coupling magnetizing flux generated, along the $d$-axis, by the components (along the same axis) of the stator and the rotor currents

- $\phi_{d 0}, \phi_{q 0}$ are additional terms whose values depend on the way the inductive parameters are defined

It is worth noticing that the coefficients $M_{d}, M_{q}$ and $M_{d q}$ are not uniquely defined. A judicious definition, suggested in [5], consists in choosing them so that, when differentiating (13) and (14) with respect to $i_{\mu d}$ and $i_{\mu q}$, one gets:

$$
M_{d} \stackrel{\text { def }}{=} \frac{\partial \phi_{\mu d}}{\partial i_{\mu d}} ; \quad M_{q} \stackrel{\text { def }}{=} \frac{\partial \phi_{\mu q}}{\partial i_{\mu q}} ; \quad M_{d q} \stackrel{\text { def }}{=} \frac{\partial \phi_{\mu d}}{\partial i_{\mu q}}=\frac{\partial \phi_{\mu q}}{\partial i_{\mu d}}
$$

It follows from (13) and (14) that the functions $\phi_{d 0}$ and $\phi_{q 0}$ must undergo the following differential equations:

$$
\frac{\partial \phi_{d 0}}{\partial i_{\mu d}}=-\frac{\partial M_{d}}{\partial i_{\mu d}} i_{\mu d}-\frac{\partial M_{d q}}{\partial i_{\mu d}} i_{\mu q} ; \quad \frac{\partial \phi_{q 0}}{\partial i_{\mu d}}=-\frac{\partial M_{q}}{\partial i_{\mu q}} i_{\mu q}-\frac{\partial M_{d q}}{\partial i_{\mu q}} i_{\mu d}
$$




$$
\frac{\partial \phi_{d 0}}{\partial i_{\mu q}}=-\frac{\partial M_{d}}{\partial i_{\mu q}} i_{\mu d}-\frac{\partial M_{d q}}{\partial i_{\mu q}} i_{\mu q} \quad \text { and } \quad \frac{\partial \phi_{q 0}}{\partial i_{\mu d}}=-\frac{\partial M_{q}}{\partial i_{\mu d}} i_{\mu q}-\frac{\partial M_{d q}}{\partial i_{\mu d}} i_{\mu d}
$$

On the other hand, substituting (13)-(14) in (8)-(11) leads to the following expressions for the $(d, q)$ flux coordinates:

$$
\begin{aligned}
& \phi_{s d}=l_{s} i_{s d}+M_{d} i_{\mu d}+M_{d q} i_{\mu q}+\phi_{d 0} \\
& \phi_{s q}=l_{s} i_{s q}+M_{q} i_{\mu q}+M_{d q} i_{\mu d}+\phi_{q 0} \\
& \phi_{r d}=l_{r} i_{r d}+k\left(M_{d} i_{\mu d}+M_{d q} i_{\mu q}+\phi_{d 0}\right) \\
& \phi_{r q}=l_{r} i_{r q}+k\left(M_{q} i_{\mu q}+M_{d q} i_{\mu d}+\phi_{q 0}\right)
\end{aligned}
$$

\section{Remark 2.}

i) As a consequence of the cross-saturation phenomenon, both rotor flux components $\left(\phi_{r d}, \phi_{r q}\right)$ depend on all components of both the stator and rotor currents. Contrarily, the standard modelling (proposed or used in [3], [4], [9], [10]) leads to the fact that a given flux component only depends on the current component along the same direction (e.g. $\phi_{r d}$ only depends on $i_{r d}$ and $i_{s d}$ ). This is a simplification of a more complex reality.

ii) It also follows from the cross-saturation that the parameters $M_{d}, M_{q}$ and $M_{d q}$ are time-varying as they are functions of the machine state variables. Formulas that link these parameters to the machine state variables are established in the next subsection.

iii) In the case of unsaturated machine, one has $M_{d q}=0$ and $M_{d}, M_{q}$ become constant.

Then, the resulting machine model coincides with the widely used standard model.

\section{Relation between the induction coefficients and the static magnetization parameter}

\section{IV.1 Definition of static magnetization parameter}

The nonlinear feature of the machine magnetic circuit is entirely accounted for through the magnetic characteristic $\Phi_{\mu}=\lambda\left(\mathrm{I}_{\mu}\right)$ (Subsection II.1). As, the instantaneous quantities $\phi_{\mu}$ and $\mathrm{i}_{\mu}$ are synchronous, one has the relation:

$$
\phi_{\mu}=\frac{\lambda\left(I_{\mu}\right)}{I_{\mu}} i_{\mu}
$$

The forthcoming development involves the static magnetizing parameter $m$, see [5]:

$$
m=\frac{\Phi_{\mu}}{I_{\mu}}=\frac{\lambda\left(I_{\mu}\right)}{I_{\mu}} \stackrel{\text { def }}{=} h\left(I_{\mu}\right)
$$


In view of (18), the $(d, q)$-components of the magnetizing flux can be expressed as follows:

$$
\phi_{\mu d}=m i_{\mu d}, \quad \phi_{\mu q}=m i_{\mu q}
$$

Then, the expressions (8)-(11) of the stator and rotor flux become:

$$
\begin{array}{ll}
\phi_{s d}=l_{s} i_{s d}+m i_{\mu d}, & \phi_{s q}=l_{s} i_{s q}+m i_{\mu q} \\
\phi_{r d}=l_{r} i_{r d}+k m i_{\mu d}, & \phi_{r q}=l_{r} i_{r q}+k m i_{\mu q}
\end{array}
$$

More generally, it is shown in subsequent sections, that all machine parameters, including induction coefficients, can be expressed as a function of $m$. But first, let us rewrite $m$ as a function of the machine state variables. Indeed from (19) and (17), one gets:

$$
m=\frac{\sqrt{\phi_{\mu d}^{2}+\phi_{\mu q}^{2}}}{\lambda^{-1}\left(\sqrt{\phi_{\mu d}^{2}+\phi_{\mu d}^{2}}\right)}=\frac{\sqrt{\left(\phi_{s d}-l_{s} i_{s d}\right)^{2}+\left(\phi_{s q}-l_{s} i_{s q}\right)^{2}}}{\lambda^{-1}\left(\sqrt{\left(\phi_{s d}-l_{s} i_{s d}\right)^{2}+\left(\phi_{s q}-l_{s} i_{s q}\right)^{2}}\right)}
$$

\section{IV.2 Analytical expressions of induction coefficients}

The nonlinearity of the magnetic characteristic implies that the induction parameters $M_{d}$, $M_{q}$ and $M_{d q}$ are varying with the state variables. As the model one develops involves such induction parameters, they need to be computable in real-time. This makes it necessary to explicitly express such parameters in terms of the machine state variables. To this end, one gets from (15) and (20) :

$$
\begin{aligned}
& M_{d}=m+\frac{\partial m}{\partial i_{\mu d}} i_{\mu d}=m+\frac{d m}{d I_{\mu}} \frac{i_{\mu d}^{2}}{I_{\mu}} \\
& M_{q}=m+\frac{d m}{d I_{\mu}} \frac{i_{\mu q}^{2}}{I_{\mu}}, \quad M_{d q}=\frac{d m}{d I_{\mu}} \frac{i_{\mu d} i_{\mu q}}{I_{\mu}}
\end{aligned}
$$

using the fact that $I_{\mu}^{2}=i_{\mu d}^{2}+i_{\mu q}^{2}$. Now, let us build up mathematical expressions that explicitly link the induction coefficients $M_{d}, M_{q}$ and $M_{d q}$ to the state variables. Equations (23)-(24) show that this objective can be reached by expressing $m$ and $d m / d I_{\mu}$ in terms of the machine state variables (measured or observed). First, recall that a set of experimental points $\left(I_{\mu}, \Phi_{\mu}\right)=\left(I_{\mu}, \lambda\left(I_{\mu}\right)\right)$ is available. A set of experimental couples $\left(I_{\mu}, h\left(I_{\mu}\right)\right)$ can thus be readily obtained, where $h($.$) is as in (19). Using ad-hoc$ interpolation tools, the experimental couples can then be used to get a smooth polynomial approximation of the function $h($.$) . The obtained polynomial approximation for the$ considered machine, denoted $Q($.$) , is represented by Fig 3. Notice also that the function$ $Q($.) is time-derivable and bounded away from zero.

On the other hand, as expressions (23)-(24) involve the derivative $d m / d I_{\mu}$, it also has to be approximated by a polynomial, denoted $R\left(I_{\mu}\right)$. To this end, a set of points $\left(I_{\mu}, d m / d I_{\mu}\right)$ 
are first estimated from the experimental set of couples $\left(I_{\mu}, \Phi_{\mu}\right)$. A smooth interpolation $R\left(I_{\mu}\right)$ is built up applying interpolation functions to the estimated points $\left(I_{\mu}, d m / d I_{\mu}\right)$. The obtained function $R\left(I_{\mu}\right)$ is shown in Fig 4. The polynomial approximations, defined up to now are summarized in Table 2. These approximations will now be based upon to get expressions that explicitly link the induction coefficients to the state variables. To this end, it follows from (19) and (22) that:

$$
\begin{aligned}
& \Phi_{\mu}=\sqrt{\left(\phi_{s d}-l_{s} i_{s d}\right)^{2}+\left(\phi_{s q}-l_{s} i_{s q}\right)^{2}} \\
& I_{\mu}=P_{i n v}\left(\sqrt{\left(\phi_{s d}-l_{s} i_{s d}\right)^{2}+\left(\phi_{s q}-l_{s} i_{s q}\right)^{2}}\right)
\end{aligned}
$$

Therefore, combining equations (23)-(24) with (21) leads to the following expressions:

$$
\begin{aligned}
& M_{d}=Q\left(I_{\mu}\right)+R\left(I_{\mu}\right) \frac{\left(\phi_{s d}-l_{s} i_{s d}\right)^{2}}{I_{\mu} Q^{2}\left(I_{\mu}\right)} \\
& M_{q}=Q\left(I_{\mu}\right)+R\left(I_{\mu}\right) \frac{\left(\phi_{s q}-l_{s} i_{s q}\right)^{2}}{I_{\mu} Q^{2}\left(I_{\mu}\right)} \\
& M_{d q}=R\left(I_{\mu}\right) \frac{\left(\phi_{s d}-l_{s} i_{s d}\right)\left(\phi_{s q}-l_{s} i_{s q}\right)}{I_{\mu} Q^{2}\left(I_{\mu}\right)}
\end{aligned}
$$

\begin{tabular}{|c|c|c|}
\hline$I_{\mu} \approx P_{i n v}\left(\Phi_{\mu}\right)$ & $m \approx Q\left(I_{\mu}\right)$ & $\frac{d m}{d I_{\mu}} \approx R\left(I_{\mu}\right)$ \\
\hline
\end{tabular}

Remark 3. Equations (27)-(29) show that, unlike the standard unsaturated case, all the induction coefficients vary (with the state variables). Nevertheless, these coefficients can be computed on-line if the state variables $\left(\phi_{s}, i_{s}\right)$ are available. 
Accounting for magnetic saturation in modelling induction machines

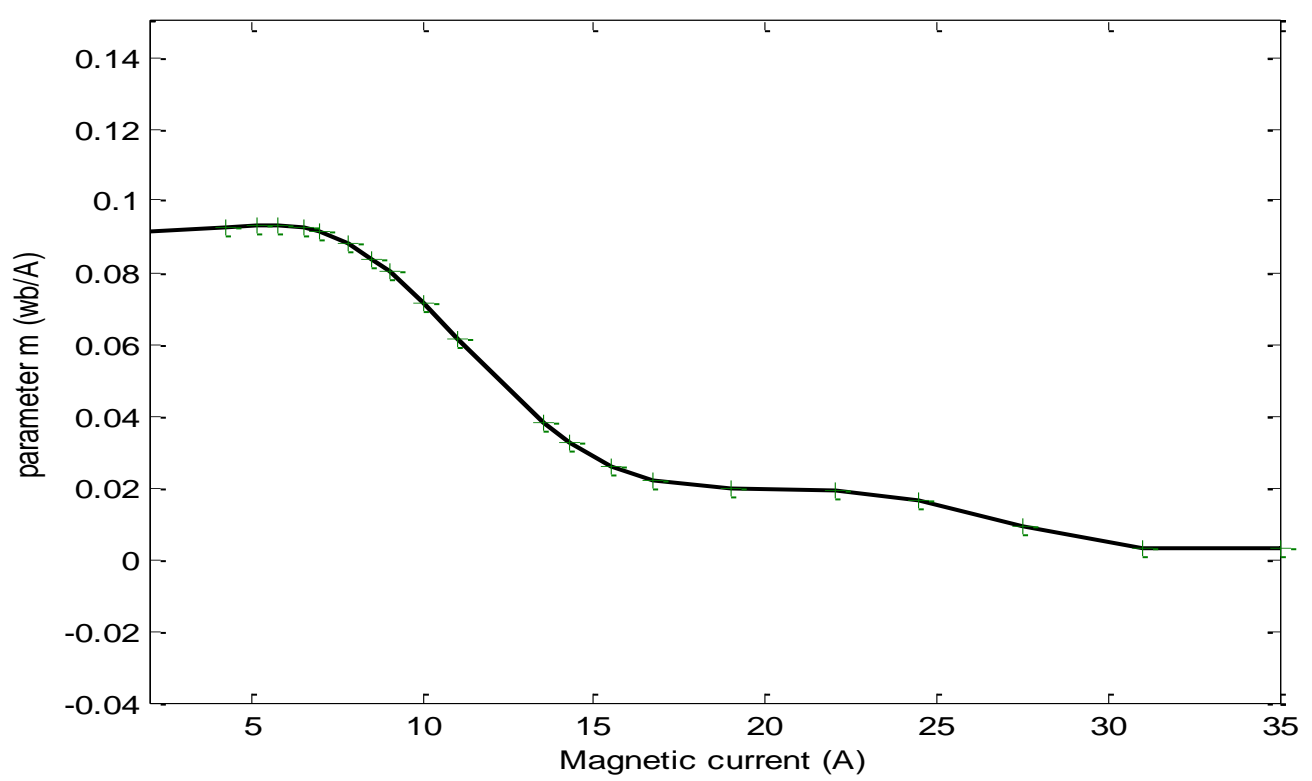

Fig 3. Experimental couples $\left(I_{\mu}, m\right)$ (crosses) and spline interpolation $Q\left(I_{\mu}\right)$ (solid). Unities: $I_{\mu}(\mathrm{A}), m$ $(\mathrm{Wb} / \mathrm{A})$

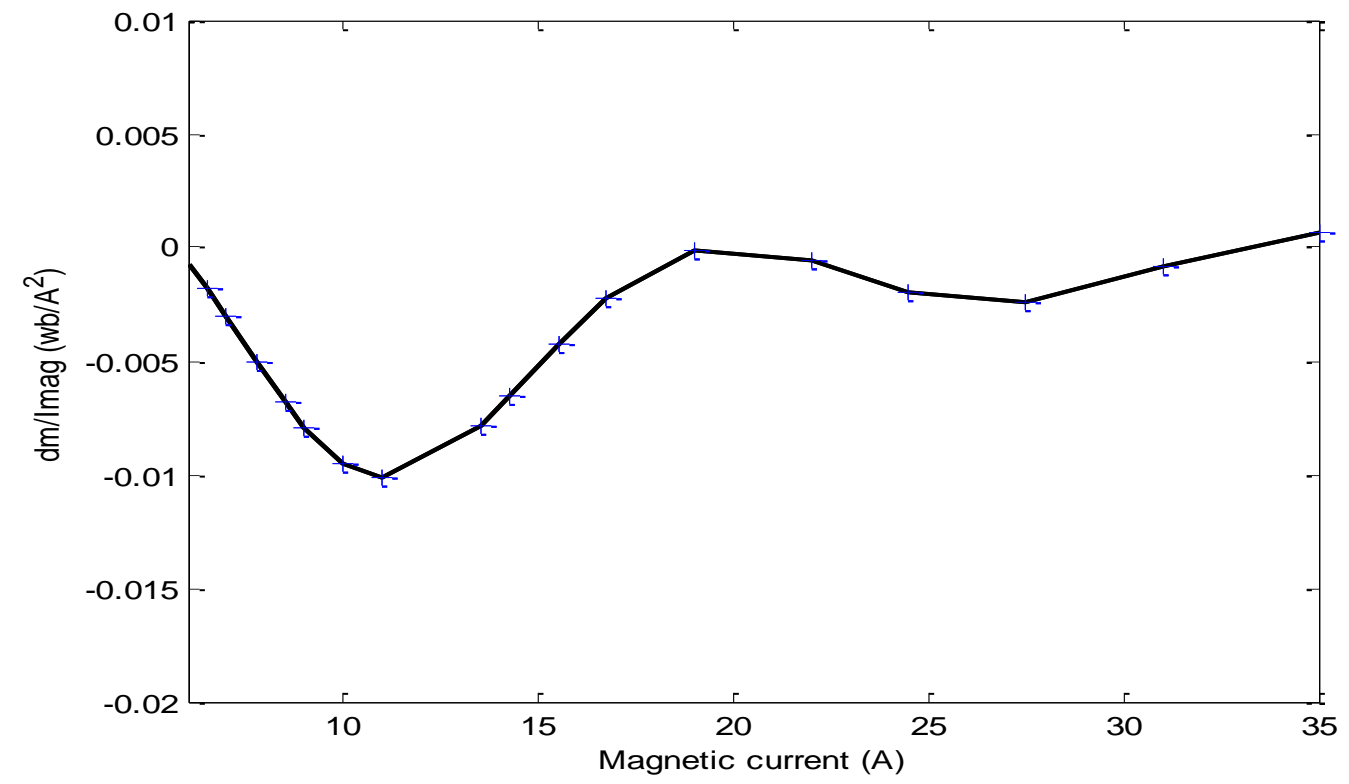

Fig 4. Experimental couples $\left(I_{\mu}, d m / d I_{\mu}\right)$ (crosses); spline interpolation $R\left(I_{\mu}\right)$ (solid). Unities: $I_{\mu}(\mathrm{A})$, $d m / d I_{\mu}\left(\mathrm{Wb} / \mathrm{A}^{2}\right)$. 


\section{Induction machine model development accounting for magnetic saturation feature}

Using the AC machine equations obtained in the previous section, the global model will now be progressively developed. Its final version must be described using only the usual state variables $X=\left[i_{s d}, i_{s q}, \phi_{r d}, \phi_{r q}, \Omega\right]^{T}$.

\section{V.1 Rotor flux equations}

From (3) one readily gets that:

$$
\frac{d \phi_{r d}}{d t}=-R_{r} i_{r d}+\omega_{r} \phi_{r q}
$$

Since $i_{r d}$ is not a state variable, it should be removed from the above equation. To this end, one obtains from (21) and (12) that:

$$
i_{r d}=\frac{\phi_{r d}-k m i_{s d}}{l_{r}+k^{2} m}
$$

which, together with (30), yields:

$$
\frac{d \phi_{r d}}{d t}=-g_{1} \phi_{r d}+g_{2} i_{s d}+\omega_{r} \phi_{r q}
$$

with $g_{1}=\frac{R_{r}}{l_{r}+k^{2} m}$ and $g_{2}=\frac{R_{r} k m}{l_{r}+k^{2} m}$. This is the first state equation. To obtain the second one, let (4) be rewritten as follows:

$$
\frac{d \phi_{r q}}{d t}=-R_{r} . i_{r q}-\omega_{r} \phi_{r d}
$$

Since $i_{r q}$ is not a state variable it should be removed from the above equation. To this end, one gets from (21) and (12) that:

$$
i_{r q}=\frac{\phi_{r q}-k m i_{s q}}{l_{r}+k^{2} m}
$$

which, together with (33), yields:

$$
\frac{d \phi_{r q}}{d t}=-g_{1} \phi_{r q}+g_{2} i_{s d}-\omega_{r} \phi_{r d}
$$

This is the second state equation.

\section{V.2 Stator current equations}

These are derived from equations (1)-(2) which are rewritten here for convenience:

$$
V_{s d}=R_{s} i_{s d}+\frac{d}{d t} \phi_{s d}-\omega_{s} \phi_{s q}
$$




$$
V_{s q}=R_{s} i_{s q}+\frac{d}{d t} \phi_{s q}+\omega_{s} \phi_{s d}
$$

As $\phi_{s d}$ and $\phi_{s q}$ are not state variables, they should be removed from the above equations. To this end, one gets from equations (21) that:

$$
\begin{aligned}
& \phi_{s d}=l_{s} i_{s d}+\frac{1}{k}\left(\phi_{r d}-l_{r} i_{r d}\right) \\
& \phi_{s q}=l_{s} i_{s q}+\frac{1}{k}\left(\phi_{r q}-l_{r} i_{r q}\right)
\end{aligned}
$$

Substituting (38)-(39) in (36)-(37), and using (31) and (34), one gets:

$$
\begin{aligned}
& V_{s d}=R_{s} i_{s d}+l_{s} \frac{d i_{s d}}{d t}+\frac{1}{k} \frac{d \phi_{r d}}{d t}-\frac{l_{r}}{k} \frac{d i_{r d}}{d t}-\omega_{s}\left(l_{s}+\frac{l_{r} m}{l_{r}+k^{2} m}\right) i_{s q}-\frac{\omega_{s} m}{k\left(l_{r}+k^{2} m\right)} \phi_{r q} \\
& V_{s q}=R_{s} i_{s q}+l_{s} \frac{d i_{s q}}{d t}+\frac{1}{k} \frac{d \phi_{r q}}{d t}-\frac{l_{r}}{k} \frac{d i_{r q}}{d t}+\omega_{s}\left(l_{s}+\frac{l_{r} m}{l_{r}+k^{2} m}\right) i_{s d}+\frac{\omega_{s} m}{k\left(l_{r}+k^{2} m\right)} \phi_{r d}
\end{aligned}
$$

In this expression, $d i_{r d} / d t$ and $d i_{r q} / d t$ must be expressed in terms of the state variables. To this end, time-derivation of the rotor flux equations (17) gives, using (16):

$$
\begin{aligned}
& \left(l_{r}+k^{2} M_{d}\right) \frac{d i_{r d}}{d t}=\frac{d \phi_{r d}}{d t}-k M_{d} \frac{d i_{s d}}{d t}-k M_{d q} \frac{d i_{s q}}{d t}-k^{2} M_{d q} \frac{d i_{r q}}{d t} \\
& \left(l_{r}+k^{2} M_{q}\right) \frac{d i_{r q}}{d t}=\frac{d \phi_{r q}}{d t}-k M_{q} \frac{d i_{s q}}{d t}-k M_{d q} \frac{d i_{s d}}{d t}-k^{2} M_{d q} \frac{d i_{r d}}{d t}
\end{aligned}
$$

Solving these equations with respect to $d i_{r d} / d t$ and $d i_{r q} / d t$ yields, using (35) and (32):

$$
\begin{aligned}
\frac{d i_{r d}}{d t}= & a_{0}\left[\frac{-R_{r}\left(l_{r}+k^{2} M_{q}\right)}{l_{r}+k^{2} m}+\omega_{r} k^{2} M_{d q}\right] \phi_{r d}+a_{0}\left[\frac{R_{r} k^{2} M_{d q}}{l_{r}+k^{2} m}+\omega_{r}\left(l_{r}+k^{2} M_{q}\right)\right] \phi_{r q} \\
& -a_{0}\left[\frac{R_{r} m k^{3} M_{d q}}{l_{r}+k^{2} m}\right] i_{s q}-a_{0}\left[\frac{R_{r} k m\left(l_{r}+k^{2} M_{q}\right)}{l_{r}+k^{2} m}\right] i_{s d} \\
& -a_{0}\left[k M_{d}\left(l_{r}+k^{2} M_{q}\right)-k^{2} M_{d q}^{2}\right] \frac{d i_{s d}}{d t}-a_{0}\left[l_{r} k M_{d q}\right] \frac{d i_{s q}}{d t}
\end{aligned}
$$

with $a_{0}=\left(\left(l_{r}+k^{2} M_{q}\right)\left(l_{r}+k^{2} M_{q}\right)-k^{4} M_{d q}^{2}\right)^{-1}$. Substituting (43) and (32) in (40), the expression of stator voltage along the $d$ axis becomes:

$$
\begin{aligned}
v_{s d}= & \left(a_{1}+a_{1}^{\prime}\right) i_{s d}-\left(a_{2}+a_{2}^{\prime \prime}+\omega_{r} a_{2}^{\prime}\right) \phi_{r d}-\left(a_{3}-\omega_{r} a_{3}^{\prime}+\omega_{s} a_{3}^{\prime \prime}\right) \phi_{r q} \\
& +\left(a_{4}-\omega_{s} a_{4}^{\prime}\right) i_{s q}+a_{5} \frac{d i_{s d}}{d t}+a_{0} a_{6} \frac{d i_{s q}}{d t}
\end{aligned}
$$

where the meaning of the different parameters is given in Table 3. 


\begin{tabular}{|c|c|}
\hline \multicolumn{2}{|c|}{ Table 3. Parameters newly introduced in equations (43)-(44) } \\
\hline$a_{0}=\left(\left(l_{r}+k^{2} M_{q}\right)\left(l_{r}+k^{2} M_{d}\right)-k^{4} M_{d q}^{2}\right)^{-1}$ & $a_{l}=a_{0} \frac{R_{r} l_{r} m\left(l_{r}+k^{2} M_{q}\right)}{l_{r}+k^{2} m}$ \\
\hline$a_{5}=l_{s}+a_{0} l_{r}\left(M_{d}\left(l_{r}+k^{2} M_{q}\right)-k^{2} M_{d q}^{2}\right)$ & $a_{l}^{\prime}=R_{s}+\frac{R_{r} m}{l_{r}+k^{2} m}$ \\
\hline$a_{2}=-a_{0} \frac{R_{r} l_{r}\left(l_{r}+k^{2} M_{q}\right)}{k\left(l_{r}+k^{2} m\right)}, \quad a_{4}^{\prime}=l_{s}+\frac{l_{r} m}{l_{r}+k^{2} m}$ & $a_{4}=a_{0} \frac{l_{r} R_{r} k^{2} m M_{d q}}{\left(l_{r}+k^{2} m\right)}$ \\
\hline$a_{3}^{\prime \prime}=\frac{k m}{l_{r}+k^{2} m}$ & $a_{2}^{\prime \prime}=\frac{R_{r}}{k\left(l_{r}+k^{2} m\right)}$ \\
\hline$a_{3}=a_{0} \frac{l_{r} R_{r} k M_{d q}}{\left(l_{r}+k^{2} m\right)}$ & $a_{3}^{\prime}=\frac{1}{k}\left(1-a_{0} l_{r}\left(l_{r}+k^{2} M_{q}\right)\right.$ \\
\hline
\end{tabular}

Operating similar transformations on the stator voltage along the $q$ axis, one gets:

$$
\begin{aligned}
v_{s q}= & \left(a_{1}^{\prime}+b_{1}\right) i_{s q}+\left(a_{4}-\omega_{s} a_{4}^{\prime}\right) i_{s d}+\left(b_{2}-a_{2}^{\prime \prime}+\omega_{r} a_{2}^{\prime}\right) \phi_{r q} \\
& +\left(-a_{3}-\omega_{r} b_{3}+\omega_{s} a_{3}^{\prime \prime}\right) \phi_{r d}+b_{5} \frac{d i_{s q}}{d t}+a_{6} \frac{d i_{s d}}{d t}
\end{aligned}
$$

where the newly introduced coefficients are defined in Table 4.

$$
\begin{array}{cc}
\text { Table 4. Parameters newly introduced in equation (45) } \\
b_{1}=a_{0} \frac{R_{r} l_{r} m\left(l_{r}+k^{2} M_{d}\right)}{l_{r}+k^{2} m} & b_{2}=a_{0} \frac{l_{r} R_{r}\left(l_{r}+k^{2} M_{d}\right)}{k\left(l_{r}+k^{2} m\right)} \\
b_{5}=l_{s}+a_{0} l_{r}\left(M_{q}\left(l_{r}+k^{2} M_{d}\right)-k^{2} M_{d q}^{2}\right) & b_{3}=k^{-1}\left(1-a_{0} l_{r}\left(l_{r}+k^{2} M_{d}\right)\right)
\end{array}
$$

Now the state equations of the stator currents can readily be obtained by solving equations (44) and (45) with respect to $d i_{s d} / d t$ and $d i_{s q} / d t$. Doing so, one gets:

$$
\begin{aligned}
\frac{d i_{s d}}{d t}= & -\left(q_{1}+q_{1}^{\prime} \omega_{s}\right) i_{s q}-\left(q_{2}+q_{2}^{\prime} \omega_{s}\right) i_{s d}-\left(q_{3}+q_{3}^{\prime} \omega_{r}+q_{3}^{\prime \prime} \omega_{s}\right) \phi_{r q} \\
& -\left(q_{4}+q_{4}^{\prime} \omega_{r}+q_{4}^{\prime \prime} \omega_{s}\right) \phi_{r d}-q_{5} v_{s d}+q_{0} v_{s q} \\
\frac{d i_{s q}}{d t}= & -\left(d_{1}+d_{1}^{\prime} \omega_{s}\right) i_{s d}-\left(d_{2}+d_{2}^{\prime} \omega_{s}\right) i_{s q}-\left(d_{3}+d_{3}^{\prime} \omega_{r}+d_{3}^{\prime \prime} \omega_{s}\right) \phi_{r d} \\
& -\left(d_{4}+d_{4}^{\prime} \omega_{r}+d_{4}^{\prime \prime} \omega_{s}\right) \phi_{q d}+d_{5} v_{s d}-d_{6} v_{s q}
\end{aligned}
$$

These equations introduce new coefficients whose meaning is described in Table 5. 


\begin{tabular}{lll}
\hline \multicolumn{3}{c}{ Table 5. Parameters newly introduced in (46)-(47) } \\
\hline$q_{0}=\frac{b_{5}}{a_{5} b_{5}-a_{6}^{2}}, \quad q_{1}^{\prime}=q_{0} \frac{b_{5} a_{4}^{\prime}}{a_{6}}, \quad q_{2}^{\prime}=q_{0} a_{4}^{\prime}$ & $q_{1}=q_{0}\left(a_{1}-b_{1}-\frac{b_{5} a_{4}}{a_{6}}\right)$ \\
$q_{2}=q_{0}\left(a_{4}-a_{6}^{-1} b_{5}\left(a_{1}^{\prime}-a_{1}\right)\right), \quad q_{4}^{\prime \prime}=q_{0} a_{3}^{\prime \prime}$ & $q_{3}=q_{0}\left(a_{2}-b_{2}+a_{6}^{-1} b_{5} a_{3}\right)$ \\
$q_{4}=q_{0}\left(-a_{0} a_{3}-\left(a_{6} a_{0}\right)^{-1} b_{5}\left(a_{1}^{\prime \prime}-a_{0} a_{2}\right)\right)$ & $q_{3}^{\prime}=q_{0}\left(a_{6} a_{0}\right)^{-1} b_{5} a_{3}^{\prime}$ \\
$q_{4}^{\prime}=q_{0} a_{6}^{-1}\left(a_{6} b_{3}-b_{5} a_{2}^{\prime}\right), \quad q_{5}=q_{0} a_{6}^{-1} b_{5}$ & $d_{2}=a_{6}^{-1}\left(a_{4}-a_{5} q_{1}\right)$ \\
$d_{1}=a_{6}^{-1}\left(a_{1}^{\prime}-a_{1}-a_{5} q_{2}\right), q_{3}^{\prime \prime}=q_{0}\left(a_{6} a_{0}\right) b_{5} a_{3}^{\prime \prime}$ & $d_{1}^{\prime}=-a_{6}^{-1} a_{5} q_{2}^{\prime}$ \\
$d_{2}^{\prime}=-a_{6}^{-1}\left(a_{4}^{\prime}-a_{5} q_{1}^{\prime}\right), d_{3}^{\prime}=a_{6}^{-1}\left(a_{2}^{\prime}+a_{5} q_{4}^{\prime}\right)$ & $d_{3}=a_{6}^{-1}\left(a_{2}^{\prime \prime}+a_{2}-a_{5} q_{4}\right)$ \\
$d_{3}^{\prime \prime}=-a_{6}^{-1} a_{5} q_{4}^{\prime \prime}, \quad d_{4}^{\prime}=-a_{6}^{-1}\left(a_{3}^{\prime}-a_{5} q_{3}^{\prime}\right)$ & $d_{4}=a_{6}^{-1}\left(a_{3}-a_{5} q_{3}\right)$ \\
$d_{4}^{\prime \prime}=-a_{6}^{-1}\left(a_{3}^{\prime \prime}-a_{5} q_{3}^{\prime \prime}\right), \quad d_{6}=q_{0} a_{5} a_{6}^{-1}$ & $d_{5}=a_{6}^{-1}\left(1+a_{5} q_{5}\right)$ \\
\hline
\end{tabular}

\section{V.3 Mechanical equation}

The mechanical power $P_{m}$ that induces the electromagnetic torque generation is given by (see e.g [7]):

$$
p_{m}=\left(\phi_{s d} i_{s q}-\phi_{s q} i_{s d}\right) \frac{d}{d t} \theta_{s}+\left(\phi_{r d} i_{r q}-\phi_{r q} i_{r d}\right) \frac{d}{d t} \theta_{r}
$$

Using the rotor currents expressions (31) and (34), in the flux equations (21) yields:

$$
p_{m}=\frac{k m}{l_{r}+k^{2} m}\left(\phi_{r d} i_{s q}-\phi_{r q} i_{s d}\right) \frac{d}{d t}\left(\theta_{s}-\theta_{r}\right)
$$

which implies that the torque is given by: $T_{e}=\frac{p k m}{l_{r}+k^{2} m}\left(\phi_{r d} i_{s q}-\phi_{r q} i_{s d}\right)$. The rotor motion equation turns out to be:

$$
\frac{d \Omega}{d t}=\frac{p k m}{J\left(l_{r}+k^{2} m\right)}\left(\phi_{r d} i_{s q}-\phi_{r q} i_{s d}\right)-\frac{T_{L}}{J}-\frac{f}{J} \Omega
$$

This is the fifth (and last) state equation. The model of a real (saturating) AC machine consists of the equations (32), (35), (46), (47) and (48), which are, for convenience, rewritten in a more condensed form:

$$
\begin{aligned}
& \dot{X}=f(X)+g(X) u \\
& y=h(X)=\left[\Omega, \phi_{r d}^{2}+\phi_{r q}^{2}\right]^{T}
\end{aligned}
$$

with : 


$$
\begin{aligned}
& X=\left[i_{s d}, i_{s q}, \phi_{r d}, \phi_{r q}, \Omega\right]^{T}, \quad u=\left[v_{s d}, v_{s q}\right]^{T}, \\
& g(X)=\left[\begin{array}{lllll}
-q_{5} & q_{0} & 0 & 0 & 0 \\
+d_{5} & -d_{6} & 0 & 0 & 0
\end{array}\right] \\
& f(X)=\left[\begin{array}{l}
-\left(q_{1}+q_{1}^{\prime} \omega_{s}\right) i_{s q}-\left(q_{2}+q_{2}^{\prime} \omega_{s}\right) i_{s d}-\left(q_{3}+q_{3}^{\prime} \omega_{r}+q_{3}^{\prime \prime} \omega_{s}\right) \phi_{r q}-\left(q_{4}+q_{4}^{\prime} \omega_{r}+q_{4}^{\prime \prime} \omega_{s}\right) \phi_{r d} \\
-\left(d_{1}+d_{1}^{\prime} \omega_{s}\right) i_{s d}-\left(d_{2}+d_{2}^{\prime} \omega_{s}\right) i_{s q}-\left(d_{3}+d_{3}^{\prime} \omega_{r}+d_{3}^{\prime \prime} \omega_{s}\right) \phi_{r d}-\left(d_{4}+d_{4}^{\prime} \omega_{r}+d_{4}^{\prime \prime} \omega_{s}\right) \phi_{r q} \\
-g_{1} \phi_{r d}+g_{2} i_{s d}+\omega_{r} \phi_{r q} \\
-g_{1} \phi_{r q}+g_{2} i_{s q}-\omega_{r} \phi_{r d} \\
\frac{p k m}{J\left(l_{r}+k^{2} m\right)}\left(\phi_{r d} i_{s q}-\phi_{r q} i_{s d}\right)-\frac{T_{L}}{J}-\frac{f}{J} \Omega
\end{array}\right]
\end{aligned}
$$

It is worth noting that, due to flux saturation, the model parameters $\left(d_{i}, d_{i}^{\prime}, d_{i}^{\prime \prime}, q_{i}, q_{i}^{\prime}, q_{i}^{\prime \prime}\right)$ are dependent on the static magnetisation parameter $m$. If the magnetic saturation is ignored, all previous parameters become constant. The proposed model then reduces to the standard model which is widely used in the control literature, e.g. [1]-[2].

\section{Experimental validation}

The experimental part of the study was performed in the Automatic Control Dept of GIPSA-Lab, using a real induction motor whose features are summarised in Table 5. The motor control inputs are the stator voltage amplitude $\left(V_{s}\right)$ and frequency $\left(\omega_{s}\right)$. The values of these control signals are imposed through a DC/AC converter. The measured variables are the stator currents, electric power and the rotor speed. The whole system is controlled by a PC through a DSP Card. The first experimental task consists in obtaining a number of experimental points of the motor magnetic characteristic. These are used to construct the spline approximation $I_{\mu}=\lambda\left(\Phi_{\mu}\right)$ (Fig 1). Presently, one proceeds with the second task

\begin{tabular}{|c|c|c|c|}
\hline \multicolumn{4}{|c|}{ Table 5 : Induction Motor Characteristics } \\
\hline Power & $P_{N}$ & 7.5 & $K W$ \\
\hline Nominal speed & $\Omega_{N}$ & 1450 & $\mathrm{Tr} / \mathrm{mn}$ \\
\hline Nominal stator voltage & $U_{s n}$ & 380 & $V$ \\
\hline Nominal stator current & $I_{s n}$ & 16 & $A$ \\
\hline Nominal flux & $\Phi_{r n}$ & 1 & $W b$ \\
\hline Nominal frequency & $f_{s}$ & 50 & $H Z$ \\
\hline Poles pair number & $p$ & 2 & \\
\hline
\end{tabular}
that consists in performing a practical validation of the newly developed model (49a-d) using real measurements. 
The experimental validation consists in comparing the responses of the model (49a-d) with experimental measures on the real motor excited by typical input voltages $V_{s}$ for different values of $\omega_{s}$.

First experiment. The model is compared with the real motor operating in the linear part of the magnetic characteristic (i.e. for small values of $\Phi_{\mu}$ and $I_{\mu}$ ). To this end, $V_{s}$ is a square signal that varies between $82 \mathrm{~V}$ and $140 \mathrm{~V}, \omega_{s}=300 \mathrm{rd} / \mathrm{s}$ and the load torque $T_{L}=0 \mathrm{Nm}$. It is worth recalling that the inputs $V_{s}$ and $\omega_{s}$ of the real machine are generated using a converter which, in addition, generates high frequency harmonics that are observed on $V_{s}$ (see Fig 6). To make the model validation more convincing, a disturbing high frequency noise is added to the input signal (Fig 6). The resulting flux norm $\left(\Phi_{r}\right)$ and current norm $\left(I_{s}\right)$ for the true machine and the model are respectively compared in Figures 7 and 8. These clearly show that the model behavior is very close to that of the real system. Fig 9 shows that the corresponding static induction parameter $m$ is almost time-invariant (this shows a relative variation of just 2\%). This is a direct consequence of the fact that the experimental conditions are such that the machine operates in the linear part of the machine magnetic characteristic. Note also that, in these conditions, the proposed model reduces to the usual standard one.

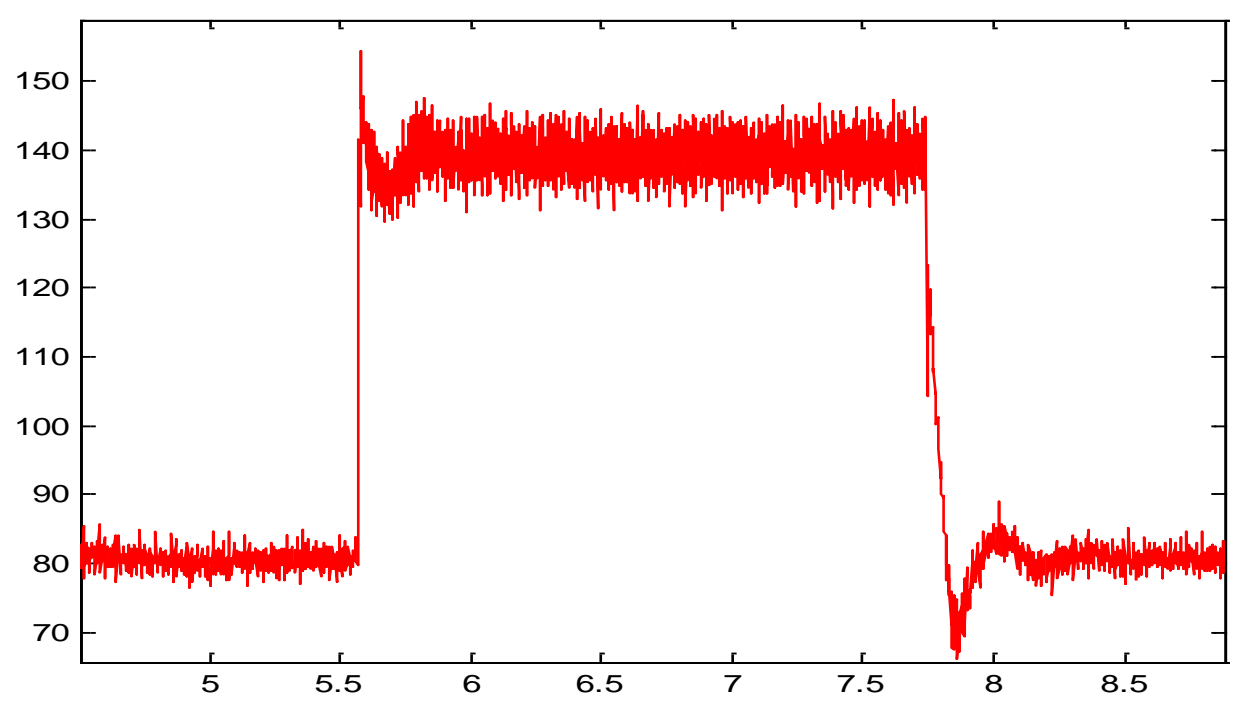

Fig 6. First experiment: the stator voltage amplitude $V s(V)$. 
F. GIRI, L. DUGARD, H. OUADI

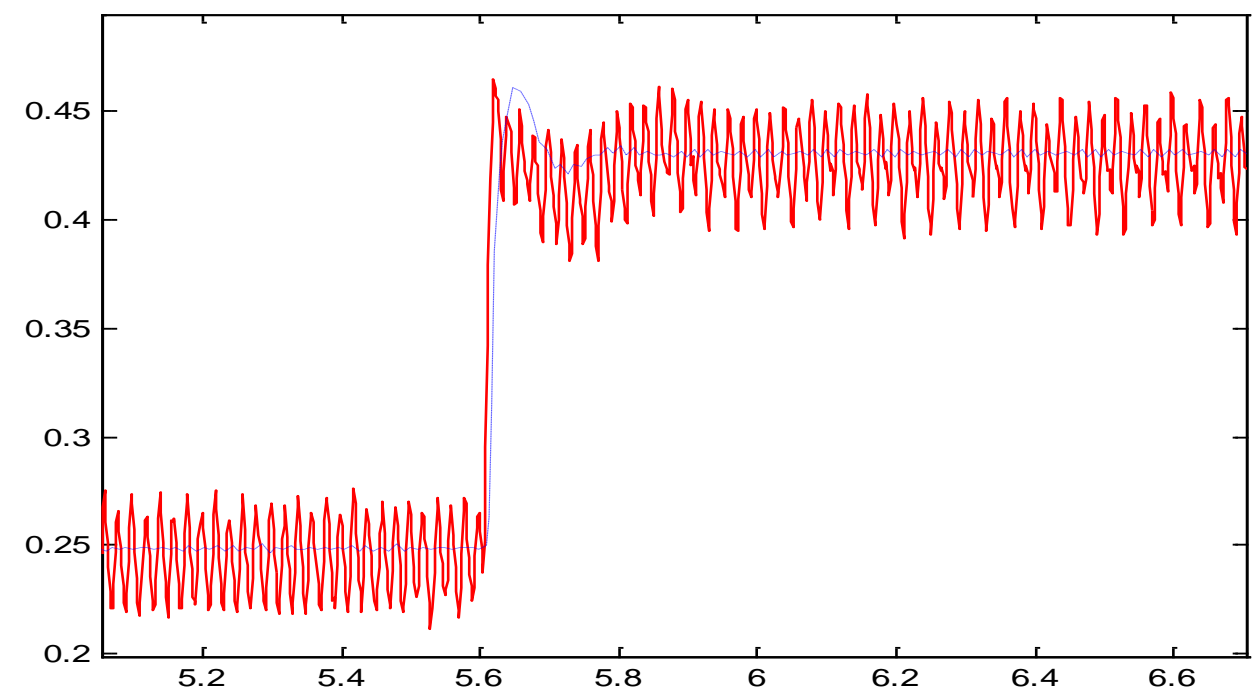

Fig 7: First experiment: flux amplitude $\Phi r(W b)$. Real machine (solid); model (49a-d) (dashed).

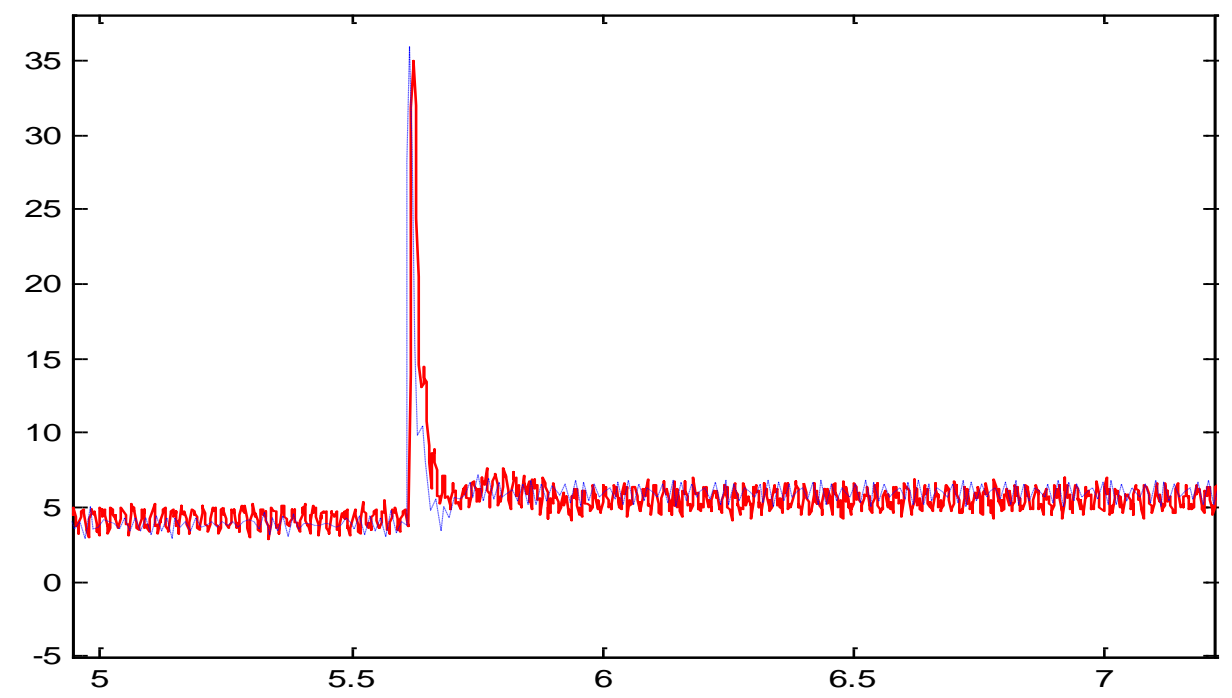

Fig 8. First experiment: the stator current amplitude $I_{s}(A)$. Real machine (solid); model (49a-d) (dashed). 


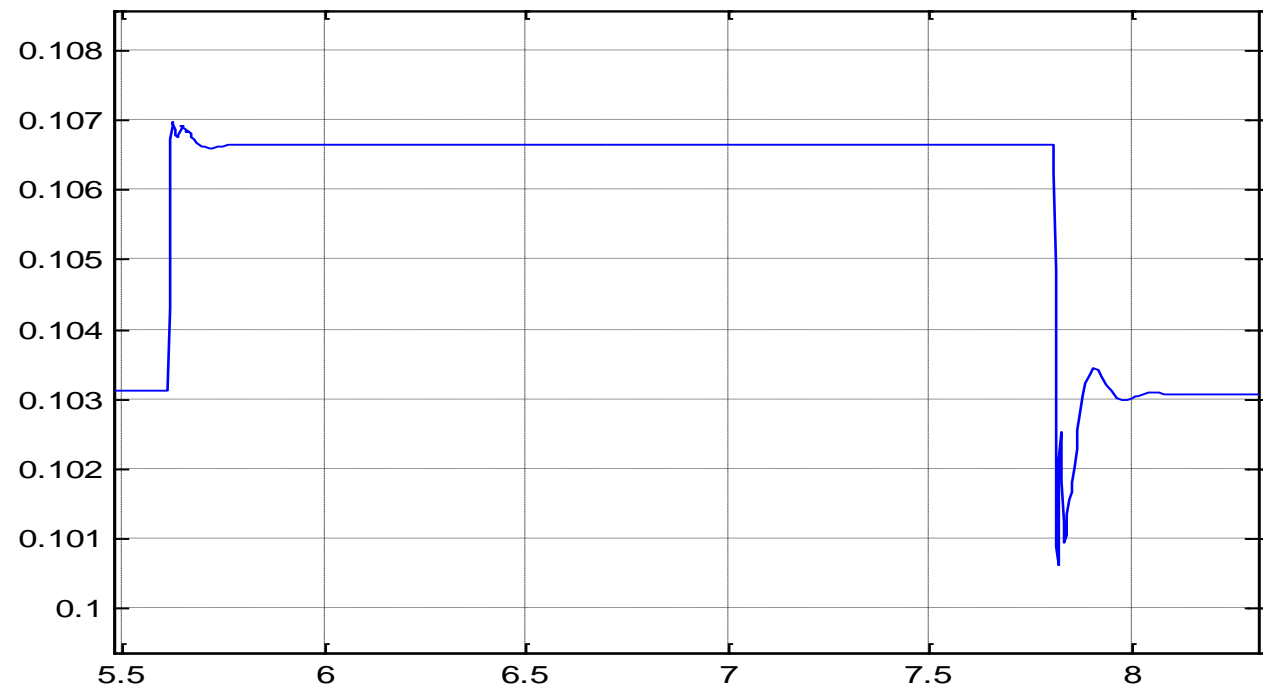

Fig 9: First experiment: static induction parameter $m(\mathrm{~Wb} / \mathrm{A})$

Second experiment. The conditions of this experiment are chosen in such a way that the machine operates in the nonlinear part of the magnetic characteristic (large values of $\Phi_{\mu}$ and $I_{\mu}$ ). To this end, the applied input voltage $V_{s}$ is a square signal switching between 220 and $265 V$ (Fig 10). The stator voltage frequency is $\omega_{s}=200(\mathrm{rd} / \mathrm{s})$ and the load $T_{L}$ is large $\left(T_{L}=30 \mathrm{Nm}\right)$. The obtained current norm $I_{s}$, for the real machine and the model, is shown in Fig 12. It is clearly seen that, again, the model is sufficiently accurate in representing the real machine. The corresponding static induction parameter $m$ is represented by Figure 13. Unlike the previous experiment, here the initial and final values of $m$ are very different (relative variation 60\%). Consequently, the proposed model cannot reduce, in these conditions, to the usual standard model. 
F. GIRI, L. DUGARD, H. OUADI

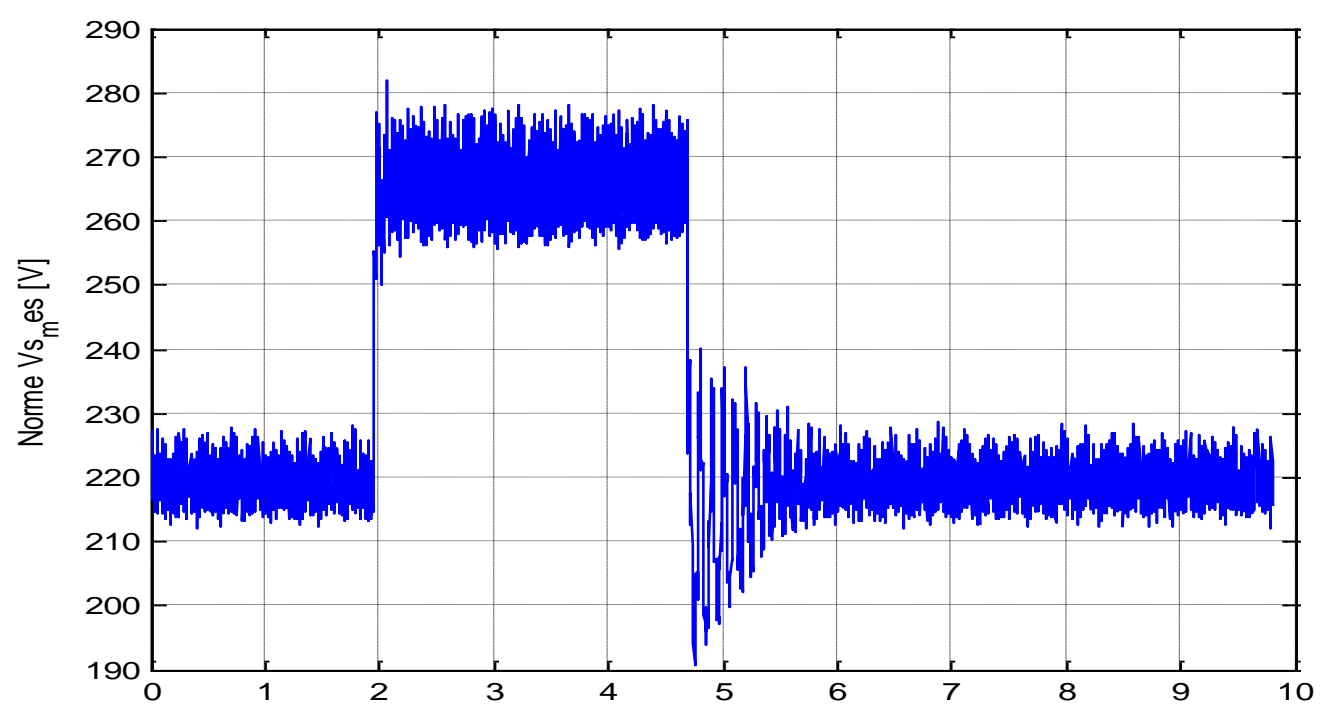

Fig 10. Second experiment: the stator voltage amplitude $V s(V)$

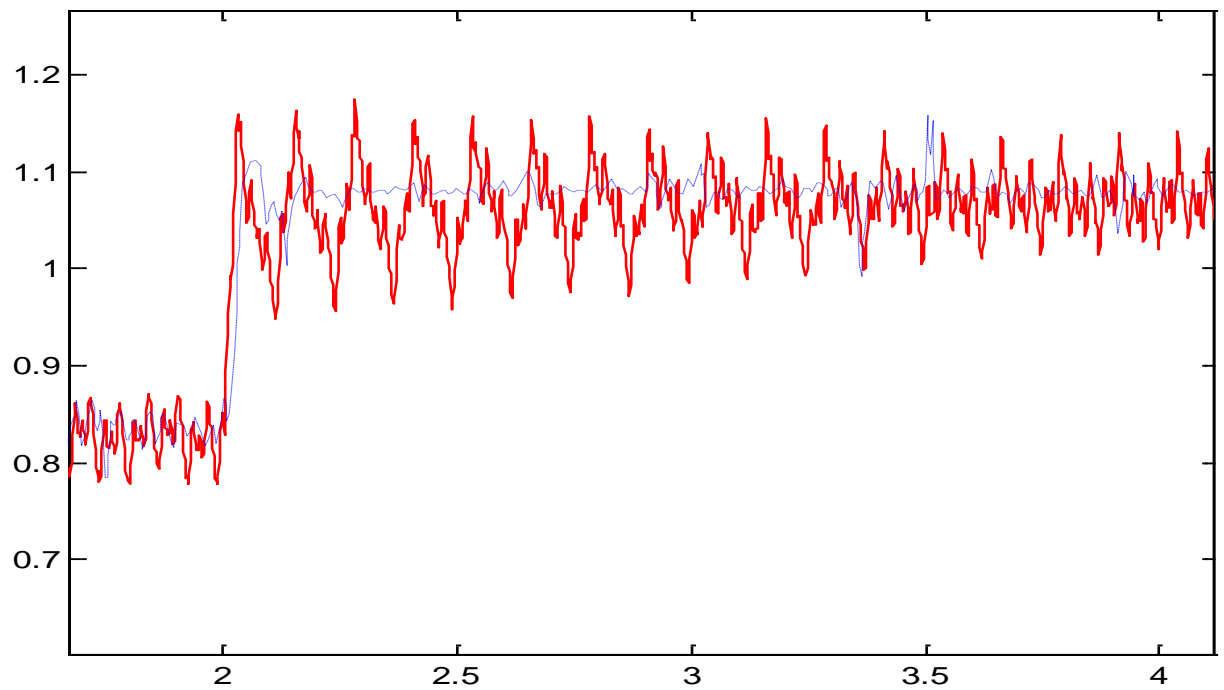

Fig 11. Second experiment: flux amplitude $\operatorname{Tr}(W b)$. Real machine (solid); model (dashed) 


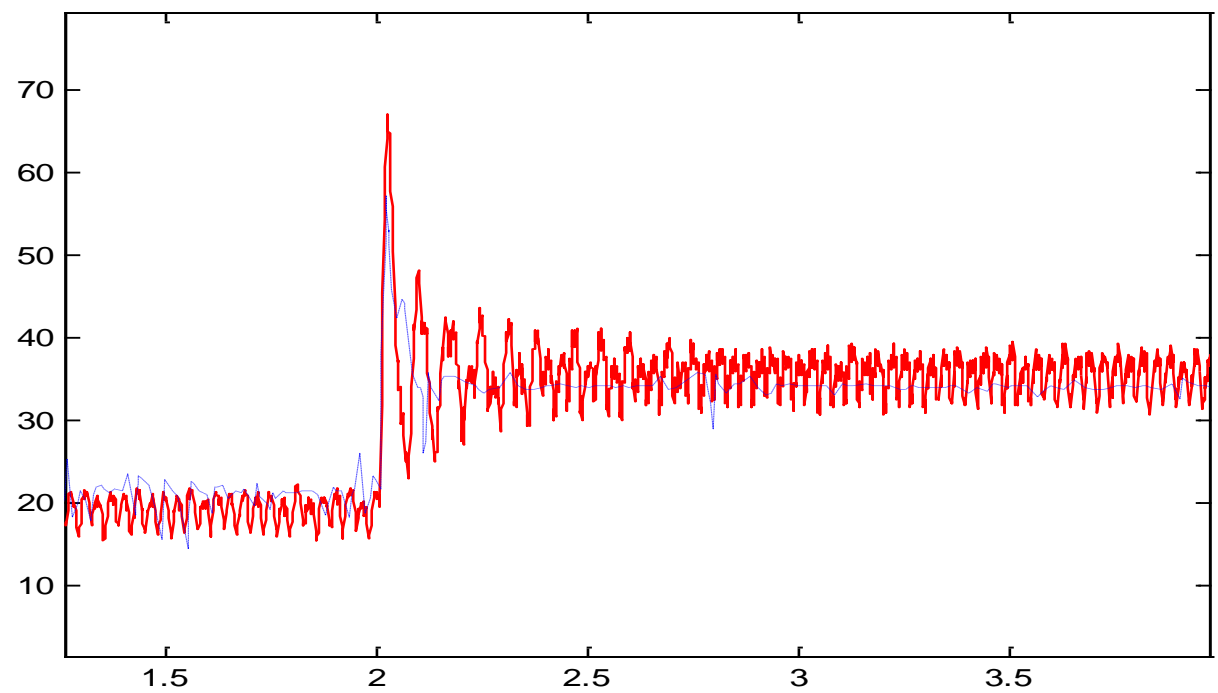

Fig 12. Second experiment: stator current norm $I_{s}(A)$. Real machine (solid); model (dashed).

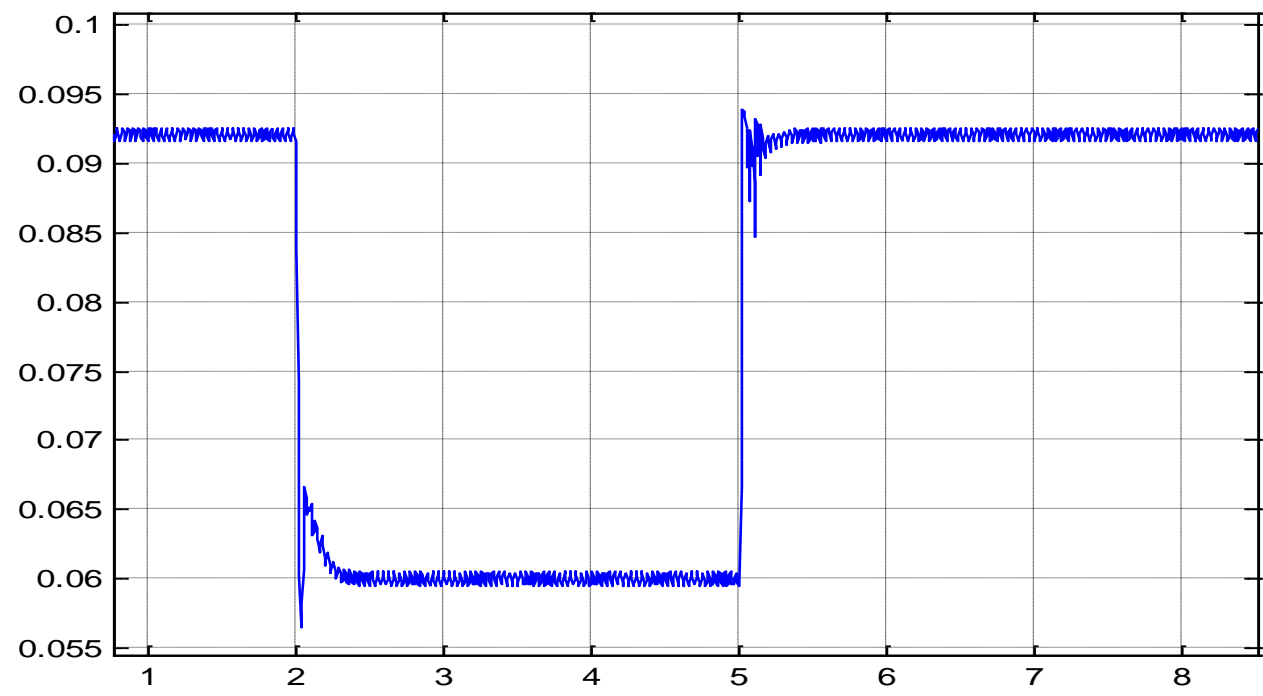

Fig 13: Second experiment: static induction parameter $m$ (in $\mathrm{Wb} / \mathrm{A}$ ) 


\section{Simplified version of the saturating model}

The new model (49a-d) was shown to be accurate through experimental validation and so it will be referred to experimentally validated model (EVM). Its accuracy makes it suitable for simulation purposes. But, it may not be quite convenient for control purpose because it involves many state dependent parameters. Therefore, it is of interest to find out if there are simplified versions that preserve the model reliability. In this section, one develops such a simplified model.

\section{1. Simplified model development}

A simplified version of the EVM can be obtained by gathering at the same side both stator and rotor leakage inductances, i.e. replacing them by a unique equivalent inductance that may be placed either at the stator side or at the rotor side, ([7], [11]). To fix idea, let $L_{\text {seq }}$ denote the equivalent leakage inductance at the stator. To this end, recall that the stator and rotor fluxes are given by equations (21). Letting $l_{r}=0$ and $l_{s}=L_{s e q}$ in (21), one gets:

$$
\begin{array}{ll}
\phi_{s d}=L_{s e q} i_{s d}+m i_{\mu d}, & \phi_{s q}=L_{s e q} i_{s q}+m i_{\mu q} \\
\phi_{r d}=k m i_{\mu d} & , \quad
\end{array}
$$

The simplified model is easily derived from model (49) by letting $l_{\mathrm{r}}=0$ and $l_{s}=L_{\mathrm{seq}}$. This gives:

$$
\begin{aligned}
& \frac{d \phi_{r d}}{d t}=-k L_{s e q} \delta \phi_{r d}+a_{1} i_{s d}+\left(\omega_{s}-\omega\right) \phi_{r q} \\
& \frac{d \phi_{r q}}{d t}=-k L_{s e q} \delta \phi_{r q}+a_{1} i_{s q}-\left(\omega_{s}-\omega\right) \phi_{r d} \\
& \frac{d i_{s d}}{d t}=\delta \phi_{r d}+a_{3} \omega \phi_{r q}-a_{2} i_{s d}+\omega_{s} i_{s q}+\frac{1}{L_{s e q}} v_{s d} \\
& \frac{d i_{s q}}{d t}=-a_{3} \omega \phi_{r d}+\delta \phi_{r q}-\omega_{s} i_{s d}-a_{2} i_{s q}+\frac{1}{L_{s e q}} v_{s q} \\
& \frac{d \Omega}{d t}=\frac{p}{J}\left(\phi_{r d} i_{s q}-\phi_{r q} i_{s d}\right)-\frac{T_{L}}{J}
\end{aligned}
$$

where:

$$
\begin{aligned}
& \delta=\frac{R_{r}}{k^{3} L_{s e q} m} \\
& a_{1}=\frac{R_{r}}{k}, \quad a_{2}=\frac{R_{s}+\frac{R_{r}}{k^{2}}}{L_{\text {seq }}}, a_{3}=\frac{1}{k L_{\text {seq }}}
\end{aligned}
$$


Remark 4. The model (52a-e) obtained by simplification is still nonlinear but it involves only one parameter (namely $\delta$ ) depending on the machine magnetic state through the magnetization coefficient. It proved to be quite convenient for designing controllers [8].

\section{2. Simplified model validation}

The responses of the simplified model defined by (52a-e) are compared with those of EVM defined by (49a-d). The applied stator voltage input $\left(V_{s}\right)$ is an amplitude-modulated sinusoid with a square modulating signal. The experimental conditions are such that the motor operates in the nonlinear part of the machine magnetic characteristic (large values of $\Phi_{\mu}$ and $I_{\mu}$ ). To this end, the stator voltage amplitude $V_{s}$ is a square signal switching between 160 and $192.5 \mathrm{~V}$ (Fig 17a), the stator current pulsation is set to $\omega_{s}=300 \mathrm{rd} / \mathrm{s}$. Figs $17 \mathrm{~b}-\mathrm{d}$ show that the responses generated by the two models are very close, except at the switching instants. That is, the simplified model is actually representative of the AC motor.

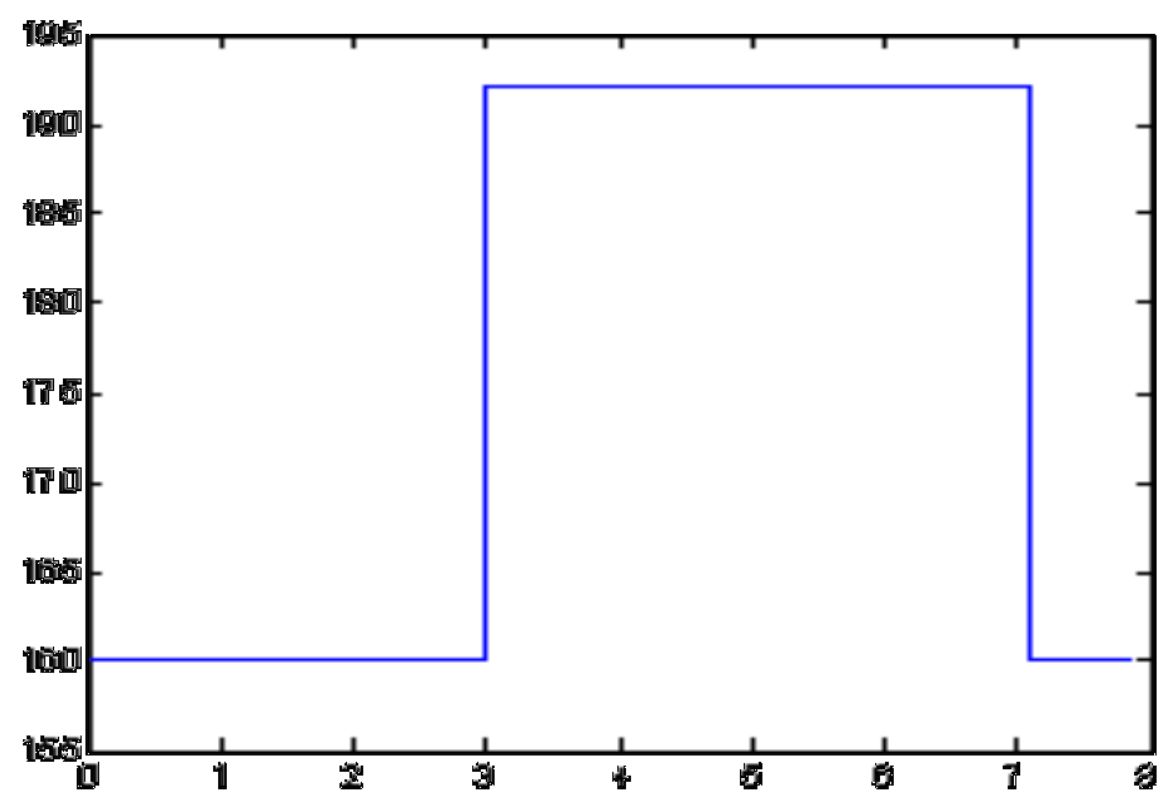

a) Stator voltage norm (in $V$ ) 
F. GIRI, L. DUGARD, H. OUADI

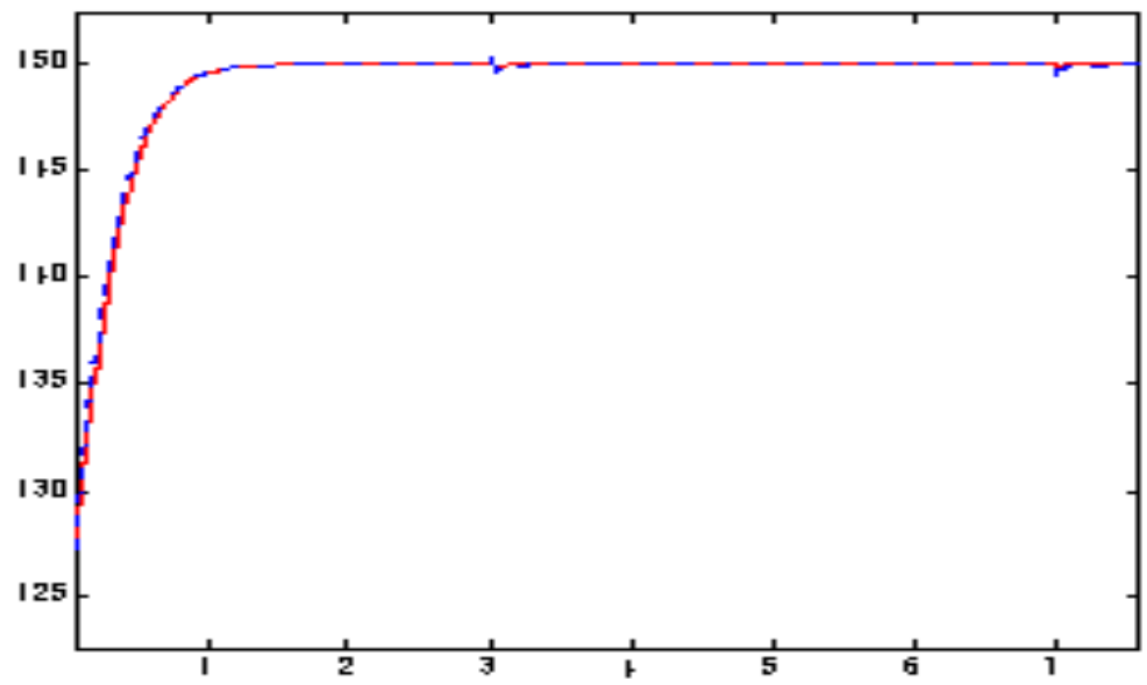

b ) Rotor speed (in $r d / s$ ): EVM (solid), simplified model (dashed)

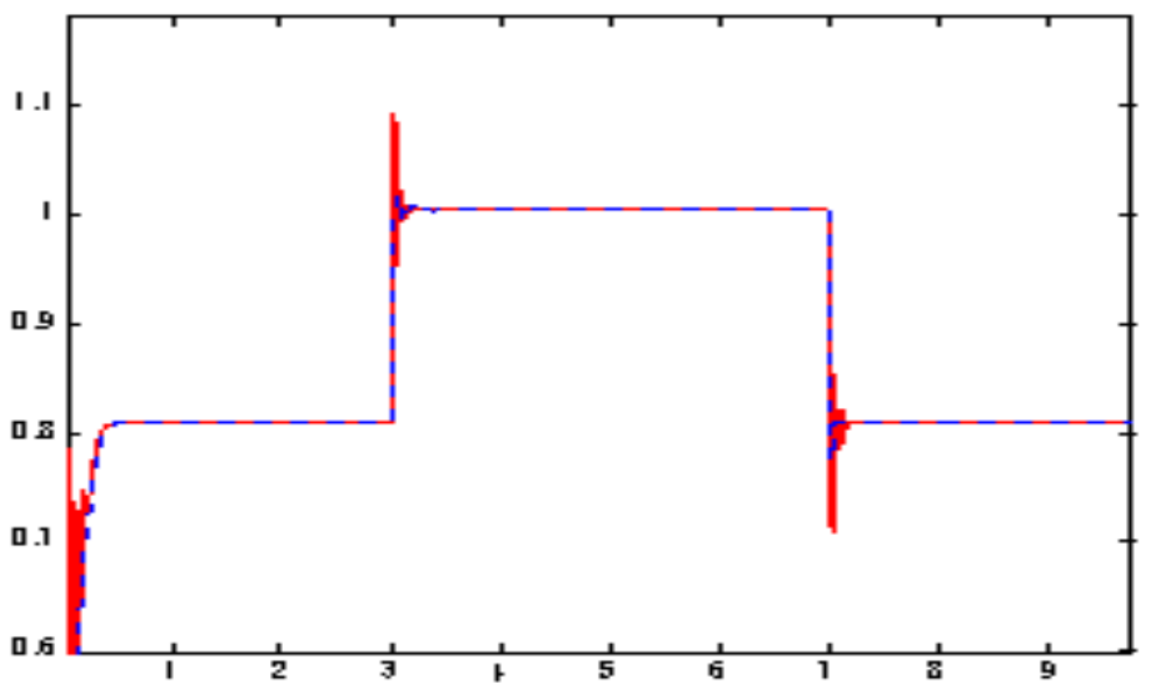

c) Rotor flux norm (in $W b$ ): EVM (solid), simplified model (dashed) 


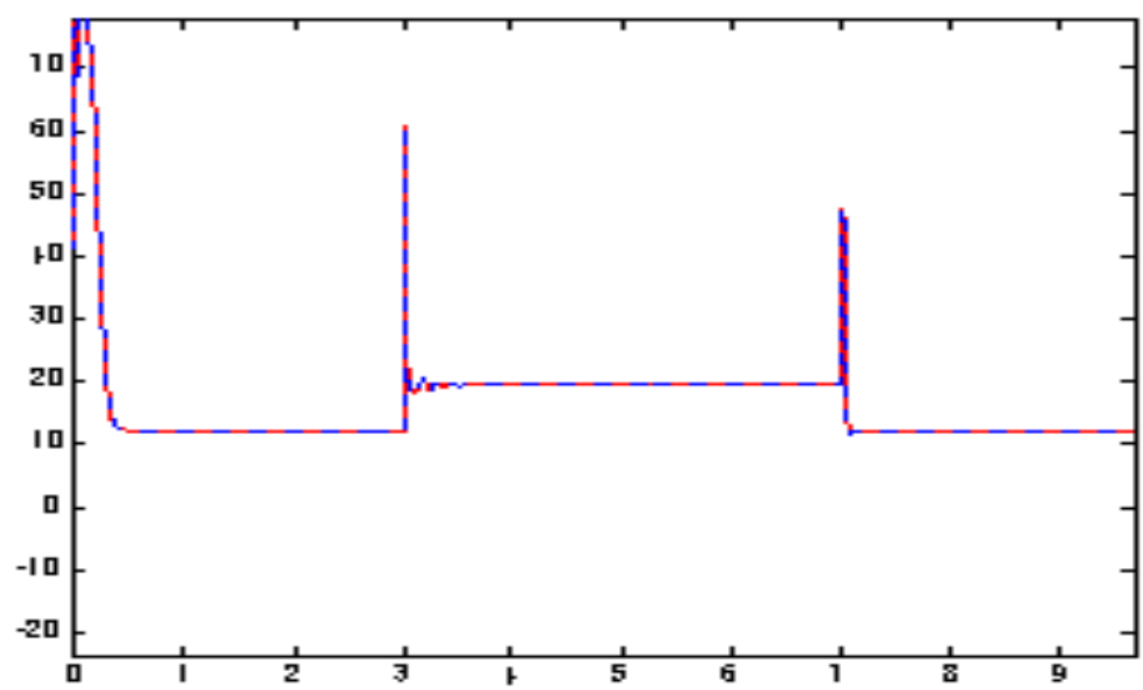

Fig 14. Responses of the EVM and simplified model (52a-e)

\section{VII.3. Supremacy of the (simplified) new model over existing models}

We have shown that the simplified model (52a-e) is a quite accurate approximation of the experimentally validated model (49a-d). It will be referred to 'simplified experimentally validated model' or simply 'SEV model' to alleviate the text. The question is whether the existing simple modelling approaches can yield equally accurate approximations. The focus is presently made on two approaches: (i) the standard modelling approach that totally ignores the magnetic saturation feature; (ii) the Heinemann-Leonhard modelling approach that accounts for such feature but it does it in an ad hoc way. Let us first present the models obtained by both approaches.

\section{VII.3.a The standard model}

As mentioned earlier (Remark 3, part iii), the standard model can be derived from the general model (49a-b) by simply letting the static induction parameter $m$ be constant. The latter is given (the quasi-constant) value obtained in the first experiment, namely $m \approx 0.1$ (see Fig 9). The obtained standard model is the following:

$$
\begin{aligned}
& \frac{d i_{s d}}{d t}=-\lambda_{s d}+\omega_{s} i_{s q}+\frac{K}{T_{r}} \phi_{r d}+K \omega \phi_{r q}+\frac{1}{\sigma_{s}} v_{s d} \\
& \frac{d i_{s q}}{d t}=-\gamma i_{s q}-\omega_{s} i_{s d}+\frac{K}{T_{r}} \phi_{r q}-K \omega \phi_{r d}+\frac{1}{\sigma_{s}} v_{s q}
\end{aligned}
$$




$$
\begin{aligned}
& \frac{d \phi_{r d}}{d t}=\frac{M}{T_{r}} i_{s d}-\frac{1}{T_{r}} \phi_{r d}+\left(\omega_{s}-\omega\right) \phi_{r q} \\
& \frac{d \phi_{r q}}{d t}=\frac{M}{T_{r}} i_{s q}-\frac{1}{T_{r}} \phi_{r q}-\left(\omega_{s}-\omega\right) \phi_{r d} \\
& \frac{d \Omega}{d t}=\frac{p M}{J L_{r}}\left(\phi_{r d} i_{s q}-\phi_{r q} i_{s d}\right)-\frac{T_{L}}{J}-\frac{f}{J} \Omega
\end{aligned}
$$

The involved parameters are defined in Table 7.

\begin{tabular}{ccc}
\hline \multicolumn{3}{c}{ Table 7. Parameters of the standard model } \\
\hline$L_{s}=l_{s}+M, \quad M=m$ & $L_{r}=l_{r}+M$ & $K=\frac{M}{\sigma L_{s} L}$ \\
$\sigma=1-\frac{M^{2}}{L_{s} L_{r}}$ & $\gamma=\frac{R_{s}}{\sigma L_{s}}+\frac{R_{r} M^{2}}{\sigma L_{s} L_{r}^{2}}$ & $T_{r}=\frac{L_{r}}{R_{r}}$ \\
\hline
\end{tabular}

\section{VII.3.b Heinemann-Leonhard saturated model}

Instead of going back to first-principles derivation (modifying it to include a saturation model of the iron) as we did in this paper, former authors chose to just add on, in a somewhat ad hoc manner, a model of magnetic saturation to standard models like (54). Following Heinemann and Leonhard [14] (see also [4]), the model (54a-e) is first subject to flux-orientation along $d$-axis. Accordingly, the $q$-axis flux equation (54d) is cancelled and the $d$-axis equation (54c) becomes:

$$
\frac{d \phi_{r d}}{d t}=\frac{M}{T_{r}}\left(-P^{-1}\left(\phi_{r d}\right)+i_{s d}\right)
$$

where $M$ is set to its nominal value that presently corresponds to the linearity region on the magnetic characteristic $P($.$) (Fig 1). Equation (55) is completed by equations (54a),$ (54b) and (54e) which remain unchanged. The resulting model is rewritten for convenience:

$$
\begin{aligned}
& \frac{d i_{s d}}{d t}=-i_{s d}+\omega_{s} i_{s q}+\frac{K}{T_{r}} \phi_{r d}+\frac{1}{\sigma_{s}} v_{s d} \\
& \frac{d i_{s q}}{d t}=-\gamma i_{s q}-\omega_{s} i_{s d}-K \omega \phi_{r d}+\frac{1}{\sigma_{s}} v_{s q} \\
& \frac{d \phi_{r d}}{d t}=\frac{M}{T_{r}}\left(-P^{-1}\left(\phi_{r d}\right)+i_{s d}\right)
\end{aligned}
$$




$$
\begin{aligned}
& \frac{d \Omega}{d t}=\frac{p M}{J L_{r}} \phi_{r d} i_{s q}-\frac{T_{L}}{J}-\frac{f}{J} \Omega \\
& w_{s}=p \Omega+\frac{M}{T_{r}} \frac{i_{s q}}{\phi_{r d}}
\end{aligned}
$$

\section{VII.3.c. Limitations of the standard and Heinemann-Leonhard models}

The standard model (described by (54a-e)) and Heinemann-Leonhard (HL) model (described by (56a-e)) will now be compared using numerical simulations with the proposed SEV model (52a-e). To be meaningful, the comparison is performed in different operational conditions. Accordingly, the machine operates in both linear and nonlinear zones of its magnetic characteristic. This is achieved by modulating the stator voltage amplitude with a square signal switching between 80 and 200V (Fig 15). The stator voltage frequency is set to $\omega_{s}=100 \mathrm{rd} / \mathrm{s}$. The load couple $T_{L}$ switches between $0 \mathrm{Nm}$ and $15 \mathrm{Nm}$ (Fig 16).

Figs 17-19 show that, as long as $V s=80 \mathrm{~V}$ (time-interval [0 s, 6s]), the responses (flux and stator current norms) of the standard and HL models are very close to those of the SEV model. This is normal because the machine operates in the linearity region of its magnetic characteristic. In fact, the rotor flux level is relatively low $\left(\Phi_{r} \leq 0.6 \mathrm{~Wb}\right)$ and the static magnetization parameter $m$ (for the SEV model (52a-e) or the original model (49a-d)) is very close to the value used to obtain the standard model (i.e. $m=0.09$ ). In these conditions, the standard and HL models are equally representative of the real machine.

The situation is quite different when the machine operates in the nonlinear region of its magnetic characteristic. This is illustrated in the time interval [6 $s, 15 \mathrm{~s}]$ by setting the stator voltage to the high value $V s=200 \mathrm{~V}$ and the rotor flux to $\Phi_{r}=1.1 \mathrm{~Wb}$ a value located at the elbow region in the magnetic characteristic. The parameter $m$ turns out to be varying, deviating far from the value 0.09 (that characterizes the standard model). Then, it is not surprising that the standard model responses deviate significantly from those of the SEV model (Figs. 17-18). The responses show that the standard model underestimates the absorbed stator current but overestimates the rotor flux. The deviation of the standard model responses (with respect to those of the SEV model) is almost $65 \%$ for the current and nearly $25 \%$ for the rotor flux. Figs 17-18 also show that the responses of the HL model (56a-e) are not so different from those of the SEV model (52a-e). This is normal since the magnetic saturation is not entirely ignored in the model (as this is the case in the standard model (54a-e)). Nevertheless, the HL model is itself unable to perfectly imitate the SEV model as the difference between both models is nearly $7 \%$ for the stator currents and 5\% for the rotor flux (Figs. 17-18). Such a deviation is simply explained by the fact that the magnetic saturation is accounted for in an ad-hoc way in the HL model. Obviously, such a deviation can even be made larger by enforcing the machine to operate 
in a more saturating regime. The larger the stator voltage and rotor flux are the larger the more important the deviation of the HL model from the SEV model.

Finally, it is observed in Fig. 19 that the differences between the three models are minor as long as the speed response is concerned. Therefore, any of these three models can be based upon, to achieve a good speed regulation. However, the energetic cost will not be the same especially in presence of high load torque. As a matter of fact, the regulators obtained from the standard and HL models will absorb higher stator currents (than the new model) because they both underestimate the current responses. Obviously, the energetic cost will be higher using the standard model than with the HL model. It is worth pointing out that the energetic issue was never raised in the previous works where speed regulation was dealt with based on the standard model.

\section{Conclusion}

In this paper, a new model that accounts for magnetic saturation has been developed for (uniform air-gap) induction motors. As a consequence of the nonlinear feature of the magnetic characteristic, a coupling arises between the motor $(d, q)$-axes. The new model (49a-d) turns out to be nonlinear and involves state-dependent parameters. Its practical validation has been performed on a $7.5 \mathrm{KW}$ induction motor. Different experiments have proved that the new model is well representative of the true machine. The supremacy of the new model over the standard (54a-e) and the Heinemann-Leonhard model (56a-e) is illustrated through simulations. On the other hand, the simplified version (52a-e) proved to be quite accurate and, consequently, can be based upon in control design. 
Accounting for magnetic saturation in modelling induction machines

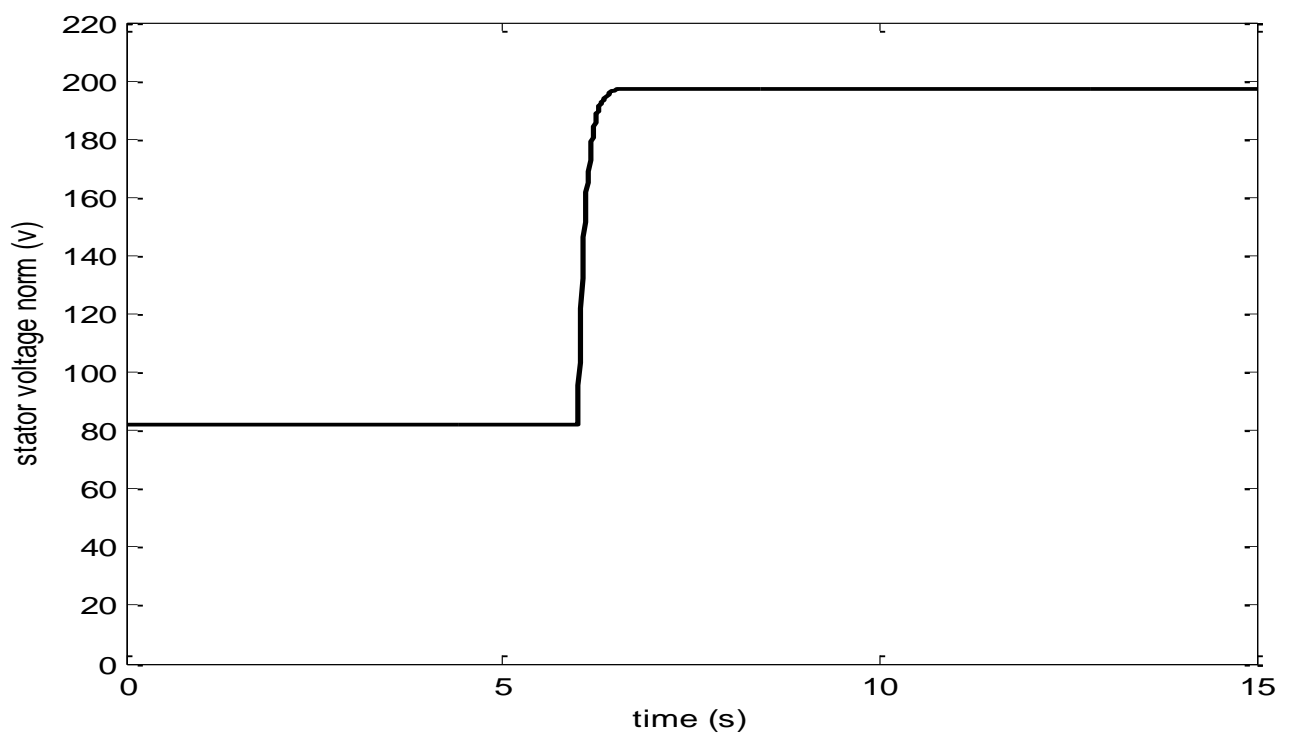

Fig. 15. Stator voltage amplitude $V_{s}(V)$

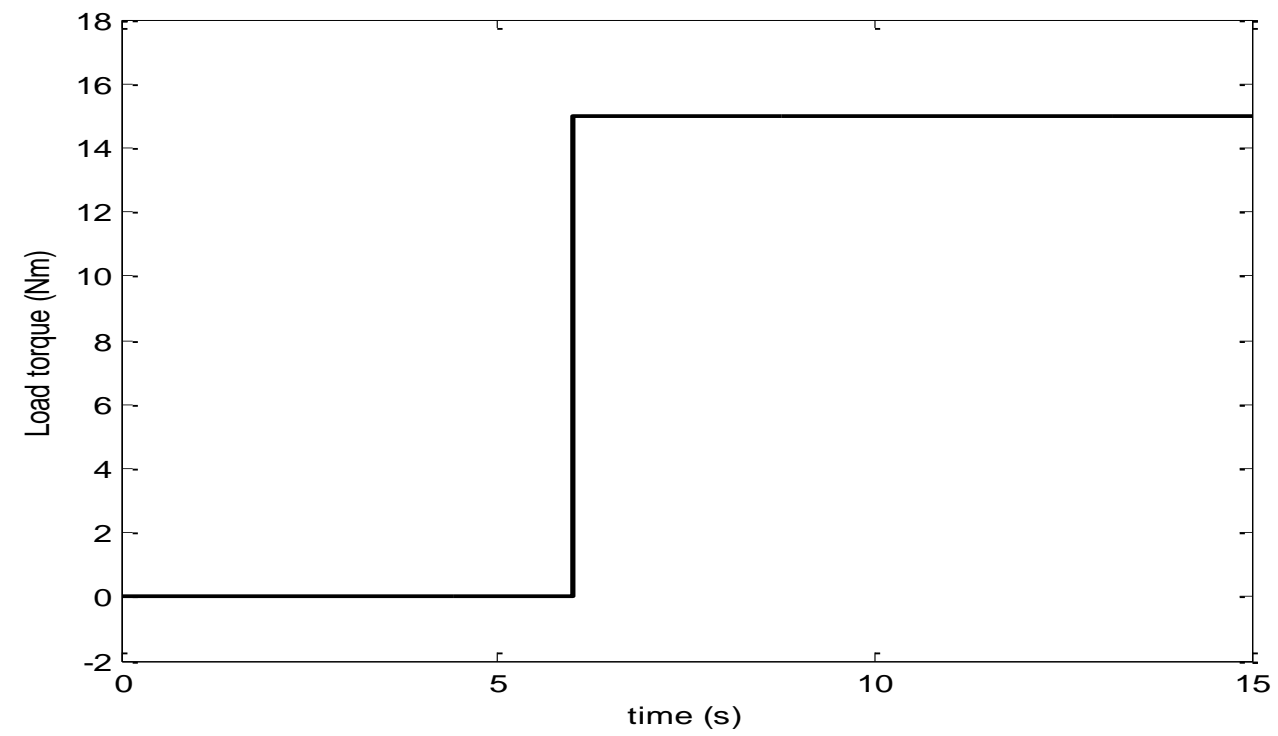

Fig. 16. Load Torque $(\mathrm{Nm})$ 
F. GIRI, L. DUGARD, H. OUADI

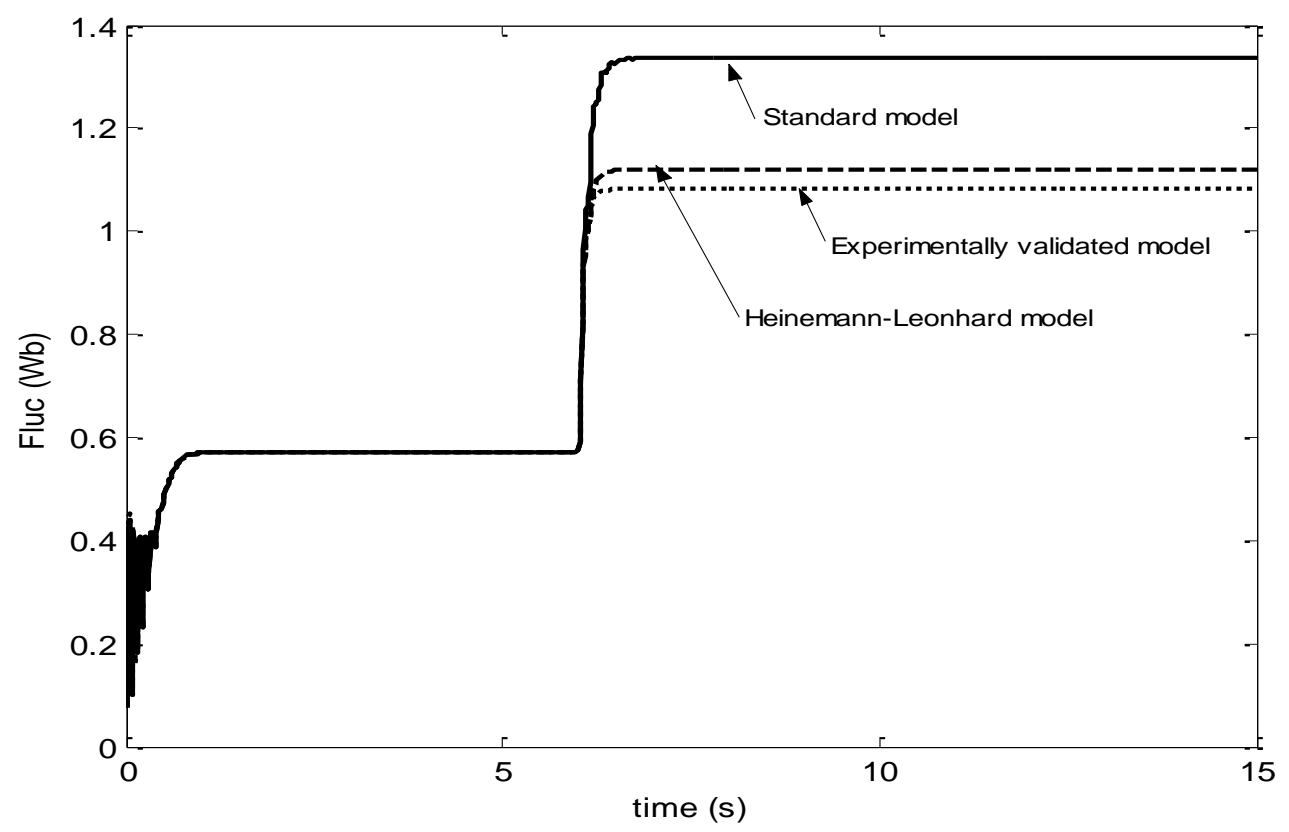

Fig 17. Rotor flux amplitude ( $W b)$ : SEV model (dotted ); Standard model (solid) and HeinemannLeonhard model (dashed)

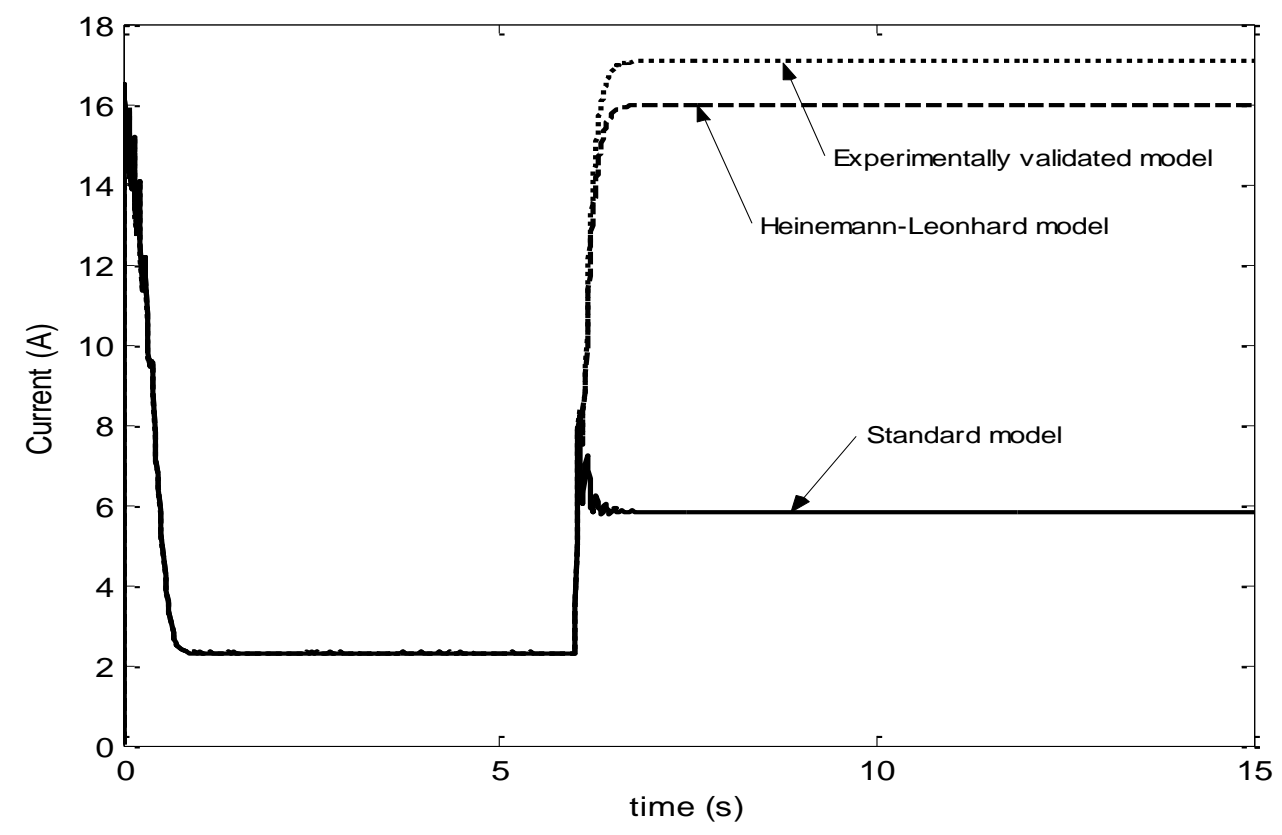

Fig 18. Stator courant amplitude (A): SEV model (dotted); Standard model (solid) and HeinemannLeonhard model (dashed) 


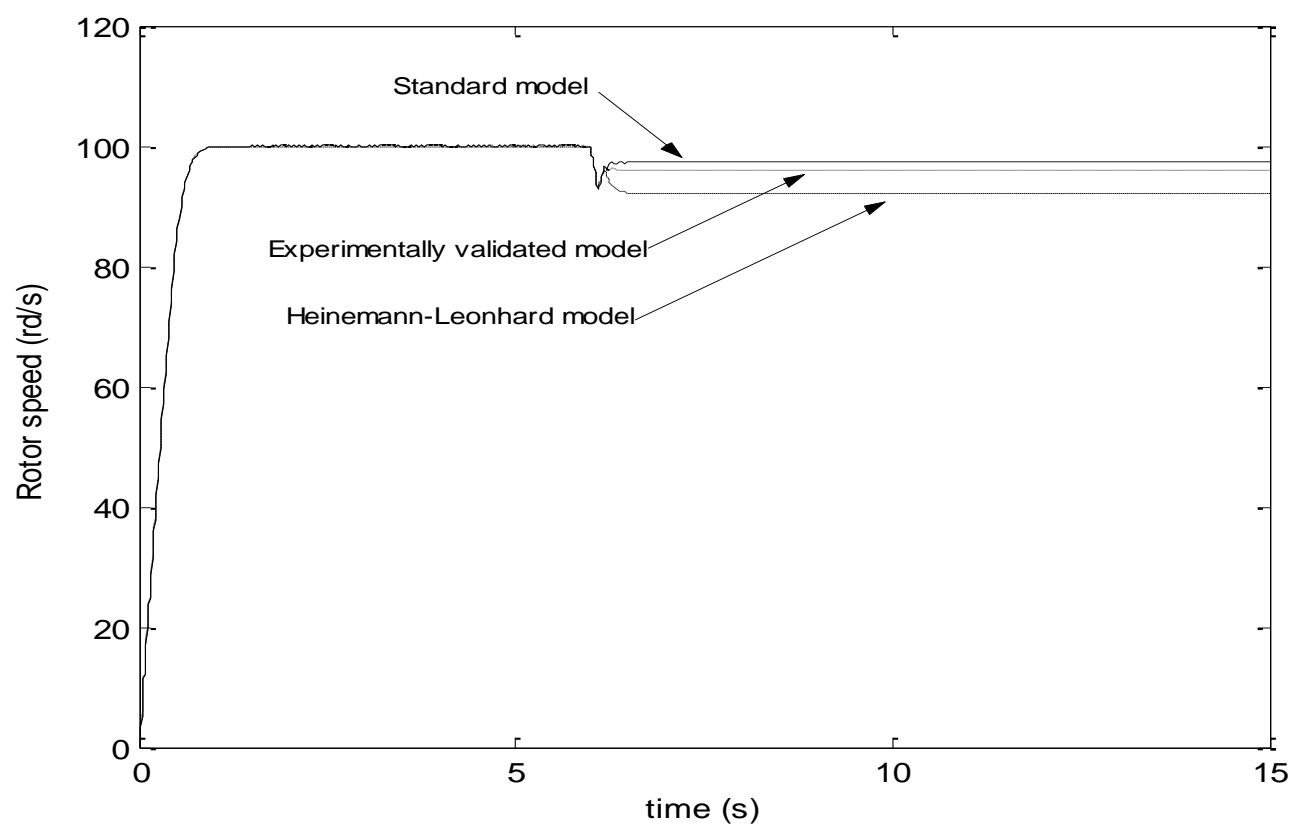

Fig 19. Rotor Speed (rd/s): SEV model (dotted); Standard model (solid) and Heinemann-Leonhard model (dashed)

\section{References}

[1] R. Ortega, P.J. Nicklasson and G. Espinosa-Perez, 'On speed control of induction motors', Automatica, Vol 32, pp. 455-460, 1996.

[2] J. Hu, M. Dawson and Z. Qu, 'Robust tracking control of an induction motor', Int. J. Robust and Nonlinear Control, Vol. 6, pp. 201-219, 1996.

[3] C.R. Sullivan and S.R. Sanders, 'Modelling the effect of magnetic saturation on electrical machine control systems', IFAC Symposium on Nonlinear Control System Design, Bordeaux, France, 1992.

[4] R.T. Novotnak, J. Chiasson, M. Bodson, 'High-performance motion control of an induction motor with magnetic saturation', IEEE Conference on Decision and Control, New Orleans, LA, pp. 2145-2150, 1995.

[5] M.S. Garrido, L. Pierrat and E. Dejaeger, 'The matrix analysis of saturated electrical machines', in J. Robert and D.K.Tran (Editors), "Modelling and simulation of electrical machines and power systems", Elsevier Science publishers B.V.NorthHolland, 1988.

[6] J.P. Caron and J.P. Hautier, 'Modelling and control of the induction machine', Edition Technip, Paris, 1995.

[7] W. Leonhard, 'Control of Electrical Drives', Springer-Verlag, New-York, 2001. 
[8] H. Ouadi. 'Modelling, observation and control of saturated induction machines'. PhD, University of Caen, 2004 (in French).

[9] J. Pedra. I. Candella and A. Barrera 'Saturation Model for squirrel cage induction motors', Electrical Power Systems Research. Vol 79, issue 7, pp 1054-1061, 2009.

[10] C.R. Sullivan and S.R. Sanders, 'Modelling the effects of magnetic saturation on electrical machine control systems', IFAC Symposium on Nonlinear Control System Design, Bordeaux, France, 1992

[11] G. Seguier and F. Notelet, 'Industrial Electronics', Editions Tec Doc, Lavoisier, Paris, 2005. ISBN 2-7430-0791-5.

[12] E. Levi, 'Impact of Cross-Saturation on Accuracy of Saturated Induction Machine Models' Transactions one Energy Conversion, Vol. 12, No 3, Sept. 1997, pp. 211-216

[13] A. Vagati, M. Pastorelli, F. Scapino and G. Franceschini. 'Impact of cross saturation in synchronous reluctance motors of the tranverse-laminated type', IEEE Transactions on industry Application, vol. IAS-36, no. 4, pp. 1039-1046, August 2000.

[14] G. Heinemann and W. Leonhard, "Self-tuning field-oriented control of an induction motor drive," in Proc. 1990 Int. Power Electron. Conf., Tokyo, Japan, Apr. 1990. 